Published in final edited form as:

Nat Genet. 2017 July ; 49(7): 1025-1034. doi:10.1038/ng.3871.

\title{
Mutations in DZIP1L, which encodes a ciliary transition zone protein, cause autosomal recessive polycystic kidney disease
}

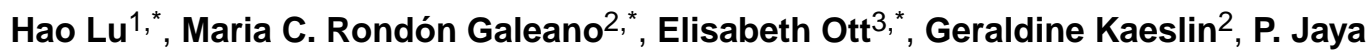 \\ Kausalya ${ }^{1}$, Carina Kramer ${ }^{3}$, Nadina Ortiz-Brüchle ${ }^{4}$, Nadescha Hilger ${ }^{4}$, Vicki Metzis ${ }^{2, \$}$, Milan \\ Hiersche $^{5}$, Shang Yew Tay ${ }^{1}$, Robert Tunningley ${ }^{6}$, Shubha Vij ${ }^{1, \&}$, Andrew D. Courtney ${ }^{2}$, \\ Belinda Whittle $^{6}$, Elke Wühl ${ }^{7}$, Udo Vester ${ }^{8}$, Björn Hartleben $^{9}$, Steffen Neuber ${ }^{5}$, Valeska \\ Frank $^{5}$, Melissa H. Little ${ }^{2,}$, Daniel Epting ${ }^{3}$, Peter Papathanasiou6,10,@ , Andrew C. \\ Perkins $^{2,11}$, Graham D. Wright ${ }^{12}$, Walter Hunziker ${ }^{1,13,14}$, Heon Yung Gee ${ }^{15,16}$, Edgar A.

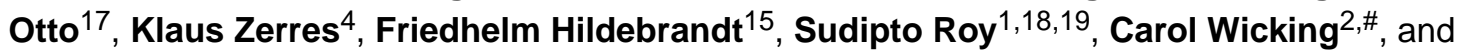 \\ Carsten Bergmann $3,4,5$,\# \\ ${ }^{1}$ Institute of Molecular and Cell Biology, Proteos, 61 Biopolis Drive, Singapore 138673, Singapore \\ ${ }^{2}$ Institute for Molecular Bioscience, The University of Queensland, St Lucia, QLD 4072, Australia \\ ${ }^{3}$ Renal Division, Department of Medicine, University of Freiburg Medical Center, Hugstetter \\ Straße 55, 79106 Freiburg, Germany \\ ${ }^{4}$ Institute of Human Genetics, RWTH Aachen University, 52074 Aachen, Germany \\ ${ }^{5}$ Center for Human Genetics, Bioscientia, 55218 Ingelheim, Germany \\ ${ }^{6}$ Australian Phenomics Facility, John Curtin School of Medical Research, Australian National \\ University, Acton ACT 2601, Australia \\ ${ }^{7}$ Division of Pediatric Nephrology, University Children's Hospital Center for Child and Adolescent \\ Medicine, Heidelberg University Hospital Heidelberg, 69120 Heidelberg, Germany \\ ${ }^{8}$ Department of Pediatric Nephrology, University Children's Hospital Essen, 45147 Essen, \\ Germany
}

Correspondence should be addressed to Sudipto Roy (sudipto@imcb.a-star.edu.sg), Carol Wicking (c.wicking@imb.uq.edu.au), or Carsten Bergmann (carsten.bergmann@bioscientia.de/ carsten.bergmann@uniklinik-freiburg.de).

\$Current address: The Francis Crick Institute, 1 Midland Road, London NW1 1AT, UK

${ }^{\&}$ Temasek Life Sciences Laboratory Limited, National University of Singapore, 1 Research Link, Singapore 117604

^Murdoch Children's Research Institute and Department of Pediatrics, University of Melbourne, Melbourne, VIC, 3052, Australia

@ Department of Materials, Imperial College London, Prince Consort Road, London SW7 2AZ, UK

${ }^{*}, \#$ These authors contributed equally

Author contributions

LH, PJK, SYT, SV, and SR performed the zebrafish mutant and morphant analysis, protein interaction studies, localization experiments with human fibroblasts and validation of PC antibodies. WH supervised the protein interaction studies. EO, CK and DE performed the zebrafish morpholino analysis. MCRG, GK, VM and ADC performed the mouse analyses, and MCRG and GK performed the localization experiments with mouse cells. RT, PP and ACP performed the screen that identified the mouse mutant, and BW was involved in mapping and identifying the mouse mutation. GDW performed the superresolution microscopy experiments. MHL assisted with design and analysis of mouse kidney experiments. NOB, NH, VF, and SN performed the human mutation analysis. $\mathrm{SN}, \mathrm{VF}, \mathrm{MH}, \mathrm{HYG}, \mathrm{EAO}, \mathrm{FH}$, and CB carried out the WES data processing and analyses. SN, VF, EW, UV, HYG, KZ, FH, and CB recruited and clinically characterized the study subjects and collected samples. BH performed histologic evaluation of the data. SR, $\mathrm{CW}$, and $\mathrm{CB}$ conceived the project, designed and supervised the experiments, analysed and interpreted the data, and wrote the manuscript. All authors reviewed the final manuscript.

Competing financial interest statement

The authors declare that there are no competing financial interests. 
9Institute of Pathology, MHH University Medical School Hannover, 30625 Hannover, Germany

${ }^{10}$ John Curtin School of Medical Research, Australian National University, Acton ACT 0200, Australia

${ }^{11}$ Mater Research Institute, Faculty of Medicine and Biomedical Sciences, University of Queensland, Woolloongabba, QLD, Australia

${ }^{12}$ Institute of Medical Biology, A*STAR, 8A Biomedical Grove, \#06-06 Immunos, Singapore 138648

${ }^{13}$ Department of Physiology, Yong Loo Lin School of Medicine, National University of Singapore, Singapore 117599

${ }^{14}$ Singapore Eye Research Institute, 20 College Road, Singapore 169856

${ }^{15}$ Department of Medicine, Boston Children's Hospital, Harvard Medical School, Boston, MA 02115, USA

${ }^{16}$ Department of Pharmacology, Brain Korea 21 PLUS Project for Medical Sciences, Yonsei University College of Medicine, Seoul, Republic of Korea

${ }^{17}$ Department of Pediatrics and Communicable Diseases, University of Michigan, Ann Arbor, Michigan 48109, USA

${ }^{18}$ Department of Pediatrics, Yong Loo Lin School of Medicine, National University of Singapore, 1E Kent Ridge Road, Singapore 119288

${ }^{19}$ Department of Biological Sciences, National University of Singapore, 14 Science Drive 4, Singapore 117543, Singapore

\section{Abstract}

Autosomal recessive polycystic kidney disease (ARPKD), usually considered to be a genetically homogeneous disease caused by mutations in PKHD1, has been associated with ciliary dysfunction. Here, we describe mutations in the DAZ interacting protein 1-like (DZIP1L) gene in patients with ARPKD, findings we have further validated by loss-of-function studies in mice and zebrafish. DZIP1L localizes to centrioles and at the distal end of basal bodies, and interacts with septin2, a protein implicated in maintenance of the periciliary diffusion barrier at the ciliary transition zone. Consistent with a defect in the diffusion barrier, we found that the ciliary membrane translocation of the PKD proteins, polycystin-1 and -2, is compromised in DZIP1L mutant cells. Together, these data provide the first conclusive evidence that ARPKD is not a homogeneous disorder, and establishes DZIPIL as a second gene involved in its pathogenesis.

\section{Keywords}

Autosomal recessive polycystic kidney disease; DZIP1L; cilia; transition zone; septin2; polycystin 


\section{INTRODUCTION}

Polycystic kidney disease (PKD) is the most common form of heritable kidney disease. In contrast to the later onset of autosomal dominant PKD (ADPKD), the autosomal recessive form (ARPKD) manifests perinatally or in childhood with enlarged kidneys ${ }^{1}$, and arterial hypertension in most surviving children ${ }^{2}$. Hepatic ductal plate malformation (DPM) can be detected histologically, but often only manifests clinically with increasing age.

In addition to ADPKD and ARPKD, cystic kidney disease is a feature of some syndromic ciliopathies, which arise from dysfunctional primary cilia ${ }^{3}$. Cilia are microtubule-based organelles that project from the surface of most vertebrate cells, and function in chemo- and mechano-sensation and fluid transport. The ciliary axoneme is anchored by the centriolederived basal body, and is ensheathed by the ciliary membrane. Located between the basal body and axoneme, the transition zone forms a diffusion barrier that modulates the selective passage of proteins to and from the cilium ${ }^{4-6}$.

PKHD1, encoding fibrocystin (FPC)/polyductin, is widely believed to be the only ARPKD gene $^{7}$. Like the two ADPKD proteins, polycystin-1 and -2 (PC1 and 2), FPC has been shown to localize to cilia ${ }^{8,9}$ and associate with the $\mathrm{PC} 1 / 2$ complex, which has long been thought to regulate calcium $\left(\mathrm{Ca}^{2+}\right)$ signalling in response to urine flow ${ }^{10-12}$. However, the role of $\mathrm{PC} 1 / 2$ complex in $\mathrm{Ca}^{2+}$ signaling and the theory that primary cilia are $\mathrm{Ca}^{2+}$ responsive mechanosensors has recently been refuted ${ }^{13-15}$. Thus, the precise mechanisms underpinning PKD remain elusive.

Here, we report the identification of a second ARPKD locus, with homozygous mutations in the cilia-related gene DZIPIL in seven patients from four families. We further validated these findings in mouse and zebrafish models. We show that DZIP1L localizes to centrioles and at the distal end of the basal body, and interacts with septin2 (SEPT2), a protein involved in maintenance of the diffusion barrier at the transition zone. Consistent with these data, we found that in DZIP1L deficient primary cilia, PC1 and PC2 show altered distribution along the ciliary membrane.

\section{RESULTS}

\section{DZIP1L is a new ARPKD gene}

In two unrelated consanguineous multiplex pedigrees (B16 and A3533) with a total of five children affected by ARPKD, mutations in known PKD genes were excluded by linkage analysis and sequencing (Fig. 1; data not shown). Genome-wide SNP analysis in DNA from parents and all affected children revealed a single overlapping homozygous peak, suggesting a new disease locus on chromosome 3q22, in a $7.5 \mathrm{Mb}$ region of genomic DNA (Fig. 1). By whole-exome sequencing (WES) and subsequent Sanger sequencing, we identified different homozygous missense DZIP1L mutations that segregated with disease in each family (Family B16: c.269C>T (p.Ala90Val); Family A3533: c.273G >C, (p.Gln91His); Accession number: NM_173543.2) (Fig. 1, Supplementary Fig. 1 and Supplementary Table 1). Interestingly, these mutations affect adjacent evolutionarily conserved residues of DZIP1L, and are bioinformatically predicted to be pathogenic (Table 1). No further convincing 
variants were identified in the candidate region in these pedigrees. All three affected individuals in family B16 showed enlarged polycystic kidneys with reduced corticomedullary differentiation, and developed arterial hypertension early during childhood. A comparable clinical course was observed in the two affected girls in family A3533 (Table 1).

Next, we analysed DZIP1L by Sanger sequencing in 218 unrelated patients with suspected ARPKD or sporadic PKD, the majority of whom had tested negative for mutations in known cystoprotein genes. A further 1330 patients with a PKD phenotype $(n=525)$ or a nephronopthisis (NPHP)-related complex ciliopathy $(n=805)$ were analyzed by nextgeneration sequencing (NGS), mainly targeted multi-gene panel testing ${ }^{16}$. Thereby, we identified different homozygous protein truncating DZIP1L mutations (c. $463 \mathrm{C}>\mathrm{T}$; (p.Gln155*) and c.1061_1062del; (p.Glu354Alafs*39)) in two additional unrelated consanguineous pedigrees, B155 and B8031, with hallmarks of ARPKD (Fig. 1, Table 1, Supplementary Fig. 1).

DZIP1L encodes a 767 amino acid (aa) protein containing a C2H2-type zinc finger motif (aa 166-189) and a series of coiled-coil domains (aa 205-406) (Supplementary Fig. 2), and previous overexpression studies showed localization to the ciliary base ${ }^{17}$. In zebrafish and mice, we and others have implicated the paralogue Dzip1 in hedgehog $(\mathrm{HH})$ signalling and ciliogenesis ${ }^{18-22}$, but there is little known about Dzip1l function in any organism.

\section{A Dzip1/ loss-of-function mouse model}

In a recessive $\mathrm{N}$-ethyl-N-nitrosourea (ENU) mutagenesis screen ${ }^{23}$, we identified a mouse mutant (warpy or wpy) with widespread embryonic dysmorphologies. Linkage analysis mapped the mutation to a $64 \mathrm{Mb}$ region on chromosome 9, and subsequent WES identified 8 homozygous mutations only one of which, a nonsense mutation in the region encoding the coiled-coil domains of Dzip11(c.1123C>T; p.Gln375*; Accession number: NM_028258.4), was within the linkage interval (Supplementary Fig. 3a). On a C57BL/6:C3H mixed or a

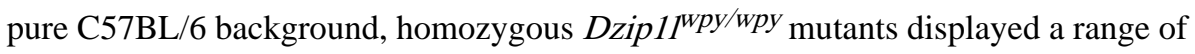
phenotypes, including highly penetrant polydactyly of all four limbs and craniofacial defects (Fig. 2), including full or partial cleft palate and bilateral cleft lip (Fig. 2f-i). Gross eye abnormalities were evident in $67 \%(n=30 / 45)$ of embryos scored from 13.5 days post coitum (dpc) on, in some cases manifesting as internalised eyes (Fig. 2j,k). Homozygous C57BL/ 6:C3H mutant embryos were recovered at roughly the expected Mendelian frequency at mid-gestation ( $23.3 \%$ at $10.5 \mathrm{dpc}, \mathrm{n}=66 / 283)$, but although some embryos were recovered up to $18.5 \mathrm{dpc}$, viability was decreased at later stages.

These phenotypes, in particular polydactyly, suggested a defect in HH signalling, a pathway regulated by the primary cilium ${ }^{24}$. Accordingly, we saw expansion of Hoxd13 and gremlin (Grem1) expression into the anterior fore- and hindlimb bud of 11.5dpc Dzip11 wpy/wpy embryos ( $\mathrm{n}=3$; Supplementary Fig. 3b; hindlimb data not shown), suggesting a decrease in the repressor form of the GLI3 transcription factor that mediates $\mathrm{HH}$ signalling in the $\operatorname{limb}^{25}$. Consistent with cilia and GLI3 regulating HH signalling downstream of ligand, the expression of sonic hedgehog (Shh) appeared unaltered in mutant limbs. Interestingly, while patched1 (Ptch1) was not consistently altered, another universal downstream marker of $\mathrm{HH}$ signalling, Gli1, showed subtle but consistent ectopic anterior expression (Supplementary 
Fig.3b). We next assessed the ability of Dzip1 $1^{w p y / w p y}$ mouse embryonic fibroblasts (MEFs) to respond to the $\mathrm{HH}$ agonist $\mathrm{SAG}$, and showed an attenuated response relative to wild-type MEFs, as measured by qRT-PCR for Gli1 (Supplementary Fig.3d). In contrast to the limb and MEFs, we saw no evidence of altered HH signalling across the dorsal-ventral axis of the neural tube in Dzip11 wpy/wpy embryos, as assessed by staining for markers of SHHdependent neuronal populations, regardless of the anterior-posterior level of the sections (n=2 for Shh, 3 for other markers; Supplementary Fig. 3c). Our data suggest tissue-specific

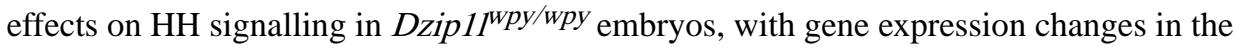
limb consistent with polydactyly.

\section{Dzip1/wpy/wpy mutants develop cystic kidney disease}

On a mixed C57BL/6:C3H background, Dzip1 $1^{\text {wpy/wpy }}$ embryos display cystic kidneys from $15.5 \mathrm{dpc}$ (data not shown). To analyse the postnatal progression of this phenotype, we crossed the mice to an outbred CD1 background, and after 4 generations, were able to recover live mice. These mice still displayed limb defects, albeit less severe than the inbred embryos, but gross facial clefting phenotypes were rare. This suggests that some of the dysmorphologies seen in the Dzip11 $1^{w p y / w p y}$ embryos, but not in ARPKD patients, may be due to genetic background effects. Homozygous mutants were born at the expected Mendelian frequency $(25.2 \%, \mathrm{n}=30 / 119)$, but showed a generalised failure to thrive, and all were sacrificed by postnatal day $(\mathrm{P}) 21$.

Histological analysis of kidney sections following at least four crosses to CD1 revealed a highly penetrant progressive cystic kidney phenotype (Fig. 3). Late in embryogenesis, the most obvious cysts were located toward the renal cortex (Fig. 3a,b; $\mathrm{n}=4$ for $15.5 \mathrm{dpc}$ ). At P0 $(n=6)$ we observed marked dilatations throughout the medullary region that stained positive for the collecting duct marker Aquaporin2 (Aqp2) (Fig. 3c-f,h,j). Some more cortical cysts stained positive for a proximal tubule marker (LTL-biotin) (Fig. 3e,f,i). The phenotype increased in severity with age, and P21 kidneys were massively cystic and larger than wildtype when corrected for body weight (Fig. 3k-m; n=3). Consistent with ARPKD in humans, we also observed subtle signs of DPM (excess of bile ducts) in the livers of Dzip1/ wpy/wpy mice at P21 (n=2) (Fig. 3n-q and Supplementary Fig. 4). Hepatic fibrosis was not detected, likely due to the young age of these mice.

\section{Inactivation of zebrafish dzip1/ causes phenotypes consistent with ciliary dysfunction}

The single orthologue of DZIP1L in the zebrafish genome (Accession number: NM_0010372277.1; Supplementary Fig. 2) is maternally expressed (Supplementary Fig. 5a), and 1 day post-fertilization (dpf) embryos express fairly ubiquitous low levels of dzip11, with a more discrete accumulation in the embryonic kidneys - the pronephric ducts (Supplementary Fig. 6a,b). Unlike the solitary primary cilium in mammalian kidney cells, zebrafish pronephric duct cells harbour motile cilia, with the mid-section of each duct containing cells bearing mono-motile cilia interspersed with multiciliated cells (MCCs). dzip1l expression was observed in the mid-section of the pronephric ducts in a pattern consistent with the MCCs (Supplementary Fig. 6b), and in the nasal placodes, another tissue populated by MCCs (Supplementary Fig. 6c). 
We next used four different antisense morpholino oligonucleotides (one translation blocking and three splice inhibitory) to knockdown dzip11 function, with all four producing largely identical cilia-related phenotypes. From 2 dpf on, dzip11 morphants presented a slight body axis curvature, hydrocephalus and defects in otolith formation in the inner ear (Supplementary Fig. 6d-g). Otolith defects were observed in 12-15\% of translation blocking $(\mathrm{n}=258)$ and splice inhibitory morpholino $(\mathrm{n}=584)$ injected embryos, but not in controls $(n=195)$ (Supplementary Fig. 6e,g,i). Despite the ciliary dysfunction phenotypes, no obvious alterations in Hh signalling were detected in 1-2 dpf dzip11 morphant embryos by in situ hybridization for $p t c 1$, eng 1 and $n k \times 2.2 b$ expression (data not shown).

To investigate defects in glomerular and pronephric tubules, we injected the dzip11 translation blocking morpholino into $T g(w t 1 b:: G F P)$ transgenic embryos that express GFP in the pronephros ${ }^{26}$. Approximately $10 \%$ of morphant embryos displayed glomerular cysts ( $\mathrm{n}=100$ ) (Supplementary Fig. 6h,i). Specificity of the dzip11 morphant phenotypes was demonstrated by co-injection of embryos with the translation blocking morpholino and wildtype dzip 11 mRNA. $85 \%$ of the morphants showed severe morphological defects, compared to $36 \%$ of the mRNA co-injected embryos (Supplementary Fig. 6j). mRNA rescue was confirmed with a second (splice blocking) morpholino (data not shown). RT-PCR analysis confirmed mis-splicing (Supplementary Fig. 5b-d).

We next generated a mutant $d z i p 11$ allele using CRISPR/Cas9 technology, targeting exons 2 and 13 with two separate guide RNAs to induce a large deletion (Supplementary Fig. 5e,f). Embryos homozygous for this deletion showed highly penetrant defects in otolith deposition, but no additional morphological abnormalities (Supplementary Fig. 5g). Since dzip1l is expressed maternally, we raised zygotic mutants to adulthood, and bred them to obtain maternal-zygotic mutant embryos. Like the zygotic mutants, maternal-zygotic dzip11 deficient embryos exhibited defective otolith deposition, but no other overt morphological changes (data not shown).

\section{DZIP1L localizes to centrioles and to the distal end of basal bodies}

To investigate the subcellular localization of endogenous DZIP1L we used two commercial antibodies, one raised to the C-terminus of DZIP1L (Sigma-Aldrich), and one to the entire protein (Abnova). Both antibodies stained centrioles and basal bodies in mouse kidney inner medullary collecting duct (IMCD3) cells, MEFs and human dermal fibroblasts (Fig. 4a-i and Supplementary Fig. 7a,c,f and data not shown). Furthermore, DZIP1L remained localized to the centrioles throughout the cell cycle, even when cilia are disassembled during mitosis (Fig. 4e-i). The mother centriole, from which the basal body is derived, acquires sub-distal and distal appendages, with distal appendages or transition fibres anchoring the basal body to the ciliary membrane at the transition zone ${ }^{27}$. DZIP1L staining overlaps with both sub-distal (ODF2) and distal (CEP164) appendage markers in IMCD3 cells (Fig. 4c,d). This suggests that DZIP1L localizes to the distal end of the mother centriole, possibly at the site where the transition zone forms. Consistent with this, DZIP1L co-localizes with the transition zone protein tectonic1 (TCTN1) in ciliated human fibroblasts (Fig. 4j). This was confirmed in retinal pigment epithelium (RPE-1) cells by superresolution microscopy (3DSIM) that improved resolution (by a factor of 2 in all dimensions; to give $\sim 120 \mathrm{~nm}$ 
resolution laterally, $x y$ ), and revealed that DZIP1L and TCTN1 indeed localize in close proximity of each other (Fig. 4k).

As expected, the C-terminal DZIP1L antibody (Sigma) did not detect specific staining in mutant Dzip11wpy/wpy MEFs or human fibroblasts from patient B155 (p.Gln155*), both of which harbour protein-truncating mutations (Supplementary Fig. 7a,b,f,g). However, some residual staining was detected with the Abnova antibody raised to the entire DZIP1L protein, in both mutant cell types (Supplementary Fig. 7c,d and data not shown). Consistent with this, qRT-PCR showed reduced but not absent Dzip11 expression in Dzip11 $1^{\text {wpy/wpy }}$ MEFs, with no compensatory change in Dzip1 (Supplementary Fig. 7e). Immunoblotting with the Abnova antibody revealed a band at the predicted size of DZIP1L in control but not B155 human cells, confirming a lack of full-length protein in mutant cells (Supplementary Fig. 7h). While we cannot rule out the possibility that the truncated mouse and human DZIP1L proteins retain some function, both would lack significant functional domains.

\section{Cilia number is largely unaffected in Dzip1/ mutant tissue}

In early passage cultures, serum-starved B155 mutant human fibroblasts displayed no obvious difference to controls in the percentage of ciliated cells, cilia morphology or localization of a number of markers of cilia and basal bodies (ARL13B, acetylated-atubulin, CEP164, DZIP1, and TCTN1; data not shown). However, in MEFs we consistently observed a decrease in the percentage of ciliated cells in serum-starved Dzip11wpy/wpy versus control cultures (Supplementary Fig. 8a). Interestingly, in a number of embryonic mouse tissues, including the limb bud mesenchyme, we saw no difference in the relative proportion of cilia between mutant and wild-type embryos ( $n=3$; Supplementary Fig. 8b). The only location we saw evidence of fewer cilia was in the collecting duct dilatations in the Dzip11wpy/wpy kidney (data not shown), but this is likely to be an effect of the dilatations rather than a cause.

We also saw no obvious primary cilia defects in dzip1l deficient zebrafish embryos (Fig. $5 \mathrm{a}, \mathrm{b}$; data not shown). However, consistent with prominent expression of dzip11 in MCCs in kidney tubules, numbers of cilia bundles were consistently reduced in morphant and zygotic and maternal-zygotic dzip11 mutant embryos (Fig. 5c-e; data not shown).

\section{DZIP1L associates with the ciliary transition zone protein SEPT2}

To further explore a function for DZIP1L at the cilium, we used human DZIP1L as bait to screen for interacting proteins in yeast two-hybrid libraries of highly ciliated human tissues (testis, lung and brain). From all three libraries, SEPT2 was identified as a high confidence interactor of DZIP1L (Supplementary Table 2). Septins are cytoskeletal GTPases, and SEPT2 functions as part of the periciliary diffusion barrier at the transition zone ${ }^{2829}$. The interaction was verified by co-immunoprecipitation of epitope-tagged DZIP1L and SEPT2 (Fig. 6a) from transfected HEK293T cells (Fig. 6b), and by co-IP of the endogenous proteins from RPE-1 cells (Fig. 6c-e). Moreover, immunostaining of human dermal fibroblasts showed co-localization of DZIP1L and SEPT2 at the ciliary base (Fig. 6f). In support of the specificity of the interaction, we were unable to detect interaction in co-IP experiments between transfected SEPT2 and several other transition zone (NPHS1, MKS1, B9D1) and 
basal body appendage (CEP89) proteins. Likewise, we failed to detect any interaction between these proteins and DZIP1L (Supplementary Fig. 9).

To localize the domains in DZIP1L that interact with SEPT2, we generated a series of epitope-tagged N- and C-terminal deletion DZIP1L constructs, and tested their ability to associate with SEPT2 in co-IP assays (Fig. 6a). We found that all, except a C-terminal fragment (from aa 500 to the end of the protein), were able to interact with SEPT2 (Fig. 6b). Intriguingly, even a small fragment consisting of the N-terminal 165 amino acids and lacking the zinc-finger and the coiled coil domains (effectively mimicking the predicted truncation of DZIP1L in patient B155), retained the ability to interact with SEPT2. This may explain why we did not observe any alteration in SEPT2 localization at the transition zone in the B155 DZIP1L mutant human fibroblasts (Fig. 6g,h).

\section{Loss of DZIP1L affects the localization of PKD proteins to the ciliary membrane}

The cystic kidney phenotype caused by loss of DZIP1L led us to investigate the ciliary localization of the two ADPKD proteins PC1 and PC2 in DZIP1L mutant cells. Using an Nterminal PC1 monoclonal antibody $(7 \mathrm{e} 12)^{30}$ (see Supplementary Fig. 10 for validation), we found that in Dzip1I wpy/wpy ciliated MEFs, PC1 was more frequently enriched at the proximal end of the axoneme compared to wild-type MEFs, which frequently showed PC1 staining along the length of the axoneme (Fig. 7a-c). In control human fibroblasts we detected PC1 at the basal body and along the axoneme, whereas there was a reduction in PC1 accumulation along the ciliary membrane of fibroblasts from individual B155 (Fig. 7df). PC2 ciliary membrane localization was similarly affected in B155 fibroblasts (polyclonal antibody to aa 349-361; see online methods for details and Supplementary Fig. 11 for validation) (Fig. 7g-i). This suggests that in the absence of correct DZIP1L function, the ciliary membrane distribution of $\mathrm{PC} 1$ and $\mathrm{PC} 2$ is compromised, possibly reflecting a defect in the barrier function of the transition zone. However, this is unlikely to be a generalized defect since the HH co-receptor smoothened (SMO) localizes normally along the axoneme in response to SAG treatment in Dzip1Iwpy/wpy MEFs (Supplementary Fig. 11). While we also analysed the localization of FPC in human fibroblasts, in our hands two independent antibodies revealed prominent staining around the basal body as described previously ${ }^{8,9}$, but not along the axoneme. This pattern remained unaffected in cells from individual B155 (data not shown).

\section{DISCUSSION}

In clinical genetics, there is a strong demand for early and reliable diagnosis of ARPKD, which is only feasible by molecular genetic analysis. To date, PKHD1 has been thought to be the only gene responsible for ARPKD. We have now identified DZIP1L mutations in ARPKD, thereby describing a second locus for this disease. While the clinical manifestations in our patients were first detected prenatally or during early childhood, none showed perinatal demise, which affects about $30 \%$ of ARPKD patients with PKHD1 mutations $^{31,32}$. Mutations in DZIP1L are a considerably rarer cause of ARPKD than PKHD1 mutations, maybe due in part to the larger size of PKHD1 relative to DZIP1L. Furthermore, our limited data suggest that the region encoding the N-terminus of DZIP1L 
may be more susceptible to mutations, a concept supported by in silico data that predict pathogenicity scores to decay towards the C-terminus (Supplementary Fig. 12). Although we have not screened large numbers of embryonically lethal patients, in view of homozygous truncating mutations in two of our patients we hypothesize that it is unlikely that $D Z I P 1 L$ mutations play a major role in this cohort. This is consistent with the birth of homozygous mutant outbred mice at expected frequencies. Despite the relatively rare occurrence of pathogenic DZIPIL mutations, ARPKD NGS diagnostic multi-gene panels should nevertheless target this gene.

Ultrasonographic patterns observed in our patients phenocopy PKHD1-linked ARPKD, with characteristic bilaterally enlarged, echogenic kidneys, poor cortico-medullary differentiation and multiple tiny cysts. Macrocysts are uncommon in infants, although they may be present with advanced clinical course. Renal failure is rarely a cause of neonatal demise in ARPKD, but renal function variably deteriorates over time. Most of our patients developed chronic renal failure during school age; end-stage renal disease (ESRD) occurred in four. However, most patients were younger than 20 years at the time of analysis, and the remaining may develop ESRD later in life. For PKHD1-linked ARPKD, 25\% of non-perinatal patients required kidney transplantation by age $32^{33}$. Most of our patients developed arterial hypertension in the first years of life, which is typical for ARPKD. DPM with congenital hepatic fibrosis is another hallmark of ARPKD. We detected subtle early signs of DPM in Dzip11 wpy/wpy mice, with the absence of more severe liver pathology, including hepatic fibrosis, likely due to the early demise of these mice. We can only speculate from our findings in the mouse that hepatic defects might also be present in our patients.

Consistent with the human phenotype, Dzip1 $11^{w p y / w p y}$ mutant mice display an early onset, progressive, cystic kidney phenotype, with cysts arising from the collecting ducts and proximal tubules. While ARPKD usually affects the collecting ducts and distal tubules, individuals may present with involvement of other nephron segments, including proximal tubules $^{34}$ (and unpublished data, CB). Moreover, a number of $P k h d 1$ mouse models display proximal tubule cysts, although the phenotype in mice carrying different alleles of $P k h d 1$ is variable, and kidney involvement generally manifests later than in Dzip1/wpy/wpy mice $35,36,37$.

Unlike patients with DZIP1L mutations, Dzip1 ${ }^{w p y / w p y ~ m o u s e ~ e m b r y o s ~ d i s p l a y ~ a ~ n u m b e r ~ o f ~}$ extrarenal dysmorphologies including limb and craniofacial defects. The amelioration of craniofacial phenotypes on crossing to an outbred background suggests that at least some of these differences may be due to genetic background, and indeed many ciliary-related phenotypes are subject to variation based on genetic modifying alleles ${ }^{38}$. It is not uncommon to see phenotypic differences between human and mouse models. For example, non-renal and hepatobiliary phenotypes have been reported in PKD mouse models, including craniofacial, cardiovascular and skeletal defects in homozygous $P k d 1$ mutant mice ${ }^{39,40}$. While we cannot exclude the involvement of DZIP1L mutations in syndromic ciliopathies, pathogenic mutations have not been detected in large cohorts of ciliopathy patients screened to date. However, our data raise the possibility that variation at DZIPIL may genetically interact with other PKD or ciliopathy loci to modify phenotype. 
Our zebrafish analysis also supports a role in the kidney, with reduced motile cilia in dzip11deficient pronephric tubules. Although the mammalian kidney is populated by primary cilia, this nevertheless suggests a conserved requirement of Dzip11 at cilia for proper kidney function across the vertebrates. It is notable that although dzip1l morphant and mutant embryos exhibited overlapping phenotypes (for example, otolith and motile cilia defects), we failed to observe body curvature and hydrocephalus in either zygotic or maternal-zygotic mutants. These effects could be morpholino-induced artefacts. However, given that all four morpholinos produced these phenotypes, it is possible that a degree of genetic compensation is operating in the mutants, as recently described ${ }^{41,42}$.

Our data suggest context or tissue-specific roles for DZIP1L in primary ciliogenesis and $\mathrm{HH}$ signalling in the mouse, whereas loss of the protein in zebrafish causes less prominent effects on these processes. Previous studies in cultured human cells suggested a redundant role for DZIP1 and DZIP1L in ciliogenesis ${ }^{17}$, a conclusion supported by our zebrafish in vivo experiments (data not shown). DZIP1 localizes to the basal body, the transition zone, and mother centriole 22,43 . Our findings reveal a similar localization pattern for DZIP1L, and proteomic studies in Chlamydomonas reinhardtii further support DZIP1L as a transition zone protein ${ }^{44}$. There is some debate over the precise boundaries between the basal body, transition zone and axoneme ${ }^{6}$, and some proteins shuttle between these structures. In keeping with a transition zone localization, we found that DZIP1L associates with SEPT2, a component of the transition zone diffusion barrier. While DZIP1L has not been rigorously associated with any of the known transition zone protein complexes, a recent mass spectroscopy study included DZIP1L amongst an extensive list of proteins pulled down by Inturned, a core component of the CPLANE transition zone complex that recruits a subset of intraflagellar transport A (IFT-A) proteins to basal bodies ${ }^{45}$. Although this raises the possibility that DZIP1L may act in the CPLANE complex, this requires further detailed analysis.

Consistent with a role for DZIP1L at the transition zone, ADPKD proteins PC1 and PC2 showed altered distribution along the ciliary membrane in the absence of correct DZIP1L function. In the renal tubules, PC1 and PC2 act in a ciliary membrane complex, and FPC has been shown to bind to and regulate PC2 ${ }^{12}$. However, we were not able to detect FPC along the ciliary axoneme to determine if this localization was also compromised by loss of DZIP1L function. In terms of how ciliary mislocalization of the polycystins may contribute to $\mathrm{PKD}$, it has been proposed that the $\mathrm{PC} 1 / 2$ complex suppresses a yet to be identified ciliadependent cyst formation signal that is inappropriately activated when the PC1/2 complex is unable to properly traffic to cilia ${ }^{46}$. Whether mislocalization of the polycystins is a major factor contributing to PKD in DZIP1L mutant mice and humans remains to be determined. Nevertheless, our data demonstrate DZIP1L dysfunction as a novel cause of ARPKD, and extend our understanding of the genetic heterogeneity underlying the pathology of this disorder. 


\section{(ONLINE) METHODS}

\section{Ethics approvals}

Human blood samples for DNA extraction and fibroblasts were obtained with written informed consent. All investigations were conducted according to the Declaration of Helsinki, and the study was approved by the Institutional Review Board of the Ethics Committee of the University Freiburg, University of Aachen, University of Michigan, Boston Children`s Hospital and collaborating institutions. All mouse experimentation was performed under approval from the Institutional Review Board of Australian National University and the relevant animal ethics committee of The University of Queensland, and conformed to ethical guidelines. All experiments involving zebrafish were approved by the animal committees of respective institutes (Regierungspräsidium Baden-Württemberg and the Singapore National Advisory Committee on Laboratory Animal Research).

\section{Data availability}

For data release, for each of the families the respective IRB-related protocols are being followed. Sequence and variant data will be deposited into $\mathrm{dbGaP}$ as a suitable public repository (https://www.ncbi.nlm.nih.gov/gap).

\section{Human haplotype analysis and SNP genome-wide linkage analysis (homozygosity mapping)}

Initially, linkage to $P K H D 1$ was excluded by haplotype analysis. Two informative flanking markers were typed in our index pedigree B16. DNA samples were available from parents and all affected individuals. Primers for PCR amplification were as published before and are available on request. Microsatellite markers were analyzed on an ABI PRISM 3130 genetic analyzer (Applied Biosystems, Darmstadt, Germany). Subsequently, we performed genomewide SNP mapping in the above first-degree consanguineous index family with three affected children (LOD score 2.4; Fig. 1) and family A3533 as reported previously ${ }^{47}$.

\section{DZIP1L mutation analysis}

Mutation analysis was done by direct sequencing for the 15 exons encoding the 767 amino acid (aa) DZIP1L protein (GenBank: NM_173543.2, NP_775814; mutation numbering corresponds to the A of the ATG-translation initiation codon in exon 2). Genomic DNA from an affected individual was amplified by PCR with oligonucleotide primers complementary to flanking intronic sequences. Primers used for PCR and direct sequencing are available on request. Samples were run and analyzed on an ABI PRISM 3130 genetic analyzer (Applied Biosystems).

In addition to conventional Sanger sequencing of DZIP1L in a total of 218 patients with suspected PKD, we performed different next-generation sequencing (NGS) based approaches. First, we used PCR-based 48.48 Access Array microfluidic technology (Fluidigm $^{\mathrm{TM}}$ ) with consecutive NGS. We applied a multiplexing approach allowing PCRbased amplification of 53 amplicons (44 exons) for 48 DNA samples simultaneously in one known and two candidate genes, including DZIPIL. A total of 96 patients with a PKDrelated phenotype were analyzed. After two rounds of amplification followed by indexing of 
all patient-derived products with 96 different 10 bp-barcodes in a subsequent PCR, $2 \times 150$ bidirectional sequencing was performed on a MiSeq platform (Illumina ${ }^{\mathrm{TM}}$ ). Second, all exons and adjacent intronic boundaries of a different number of genes (dependent on the version of our customized multi-gene panel, including DZIP1L) known or hypothesized to cause PKD and other ciliopathies were targeted by a custom SeqCap EZ choice sequence capture library (NimbleGen, Madison, Wisconsin, USA) and subsequently sequenced on a Roche 454 GS FLX or an Illumina MiSeq or HiSeq platform $(2 \times 150 \mathrm{PE})$ according to the manufacturer's protocol. A total of 1234 patients with a polycystic kidney disease phenotype $(\mathrm{n}=429)$ or an NPHP-related complex ciliopathy $(\mathrm{n}=805)$ were analyzed with an average coverage of 60-fold (GS FLX), 120-fold (MiSeq) or more than 200-fold (HiSeq), respectively. Bioinformatic analysis was performed using the Roche GS Reference Mapper $^{\mathrm{TM}}$ software (v2.6), SeqPilot SeqNext moduleTM (v3.5.2, JSI medical systems, Kippenheim, Germany) as well as an in-house bioinformatic pipeline. For all approaches, potential mutations were confirmed by Sanger sequencing and shown to segregate with the phenotype. No further mutation thought to be of pathogenic relevance for the disease phenotype was present among the patients described in this manuscript. Whole-exome sequencing (WES) and mapping of reads was carried out as previously described ${ }^{48,49}$.

\section{Mouse strains and mutation detection}

$\mathrm{N}$-ethyl-N-nitrosourea (ENU) mutagenesis screening was performed at the Australian Phenomics Facility essentially as previously described ${ }^{23}$, except that $\mathrm{C} 3 \mathrm{H}$ was used as the mapping strain. Phenotypic screening was at $14.5 \mathrm{dpc}$. The initial genome scan involved screening a pool of affected (C57BL/6xC3H) F2 mice and a pool of unaffected $\mathrm{F} 2$ mice with a set of $\sim 100$ markers that are polymorphic between C57BL/6 and C3H mice and on average span the genome at $20 \mathrm{Mb}$ intervals. The allele specific SNP markers were designed using available software to amplify a subset of validated SNPs. SNP typing was performed using the Amplifluor kit (Chemicon). The individual DNA samples from each of the affected and unaffected F2's were run with markers that showed possible linkage to the mutation. Following this approach, the wpy mutation was mapped to a $64 \mathrm{Mb}$ region on the mouse genome (GRCm38) on Chr 9 between $\underline{48804027}$ (rs3023210) and 112866211 (rs3679771). In this interval there are $>900$ genes (GRCm38). Eight genes were chosen as potential candidates and subjected to Sanger sequencing of all candidate exons $+/-15 \mathrm{bp}$, used an Applied Biosystems 3730xl capillary sequencer, using Big Dye Terminator (BDT) chemistry version 3.1 (Applied Biosytems). The raw trace files were analysed using Lasergene software (DNAstar) against the C57BL/6 mouse reference genome (NCBI). One mutation was identified in intron 19-20 of Kif9 102bp distal to exon 19 (T to C), however was unlikely to be causal. Subsequent whole exome sequencing, as previously described ${ }^{50}$, was performed on samples enriched for exonic sequences by Nimblegen solution-based capture technologies, using the Illumina GAIIx system employing 100bp end reads. This resulted in the identification of 8 homozygous mutations of which only that in Dzip11 lay within the previously mapped critical interval. The mutation in Dzip11 was confirmed using affecteds and unaffecteds from the mapping screen run with an amplifluor assay designed to the Dzip11SNV, and also by Sanger sequencing at the Australian Genomic Research Facility. Subsequently, a PCR and restriction enzyme-based genotyping assay was developed (details available on request). 
For detailed phenotypic analysis, C57BL/6:C3H mice were subsequently crossed to C57BL/6 wild-type mice through 5 generations (total of 7 wild-type crosses from G0 mutagenised founder), promoting removal of incidental ENU-induced mutations. A pure C57BL/6 line was also maintained by reviving a frozen G1 C57BL/6 individual and crossing through 9 generations with wild-type C57BL/6 mice. The initial analysis of the Dzip11 ${ }^{\text {wpy/wpy }}$ phenotype was performed on the C57BL/6:C3H mixed background following at least 2 backcrosses, and was broadly consistent in subsequent generations and also on the C57BL/6 pure background in all generations. All data presented on the outbred CD1 background follows at least 4 crosses to CD1 (in total this line has now been crossed to CD1 through 10 generations). On all background strains the overall phenotype has segregated with the Dzip11 mutation through many meioses (with amelioration of the craniofacial phenotypes on crossing to a CD1 background). In most cases wild-type littermates were used as controls, except in those cases stated where heterozygous embryos were used (Dzip11 ${ }^{w p y / t}$ heterozygous mice display no discernible morphological differences from wild-type).

\section{Antibodies, stains and primers}

Primary antibodies: mouse anti-ARL13B and rabbit-anti-ARL13B (NeuroMab, Clone N295B/66; 1:300 dilution for immunofluorescence (IF) and ProteinTech Group. \#17711-1AP; 1:500 for IF); rabbit-anti-a-tubulin (Sigma-Aldrich T5192; 1:500 for IF); rabbit-antiacetylated-a-tubulin (Cell Signalling Technology \#5335; 1:800 for IF); mouse-antiacetylated-a-tubulin (Sigma-Aldrich 6-11B-1; \#T 6793; 1:500-700 for IF); mouse-anti- $\gamma$ tubulin (Sigma-Aldrich T6557; 1:500 for IF), rabbit-anti-DZIP1L (Sigma-Aldrich \#HPA030404; 1:500 for IF); mouse-anti-DZIP1L (Abnova-MaxPab H00199221-B01P; 1:500 for IF; 1:1000 for western blot); rabbit-anti-DZIP1 (Proteintech Group, \#13779-1-AP; 1:500 for IF); rabbit-anti-SEPT2 (Sigma-Aldrich, \#HPA018481;1:2000 for western blot; 1:500 for IF); rabbit-anti-CEP164 (SAB3500022; Sigma), rabbit-anti-TCTN1 (ProteinTech Group, \#15004-1-AP; 1:500 for IF); mouse-anti-Polycystin 1 (Abcam, \#AB74115; Santa Cruz Biotechnology, \# sc-130554; 1:200 for IF); rabbit-anti-Polycystin 2 (MV12; gift from R. Witzgall; 1:500 for IF); rabbit-anti-FPC (PKHD1) (hAR-Np and hAR-c2P; gift from G. $\mathrm{Wu} ; 1: 10$ and 1:20, respectively); rabbit-anti-SMO ${ }^{51}$ was a kind gift of Rajat Rohatgi (Stanford University; 1:500 for IF); rabbit-anti-IFT88 (Proteintech 13967-1-AP; 1:300 for IF); anti-Aquaporin 2 antibody (Millipore; Cat \# AB3274; 1:200 for IF); rabbit-anti- $\beta$ catenin antibody (Abcam, \#AB6302; 1:500 for IF); goat-anti-HNF3 $\beta$ (M-20) (Santa-Cruz, \#sc-6544; 1:50 for IF); goat-anti-SHH(N-19) (Santa-Cruz, \#sc-1194; 1:100 for IF); the mouse monoclonal antibodies for Isl-1 (1:200 for IF), Pax6 (1:10 for IF) and Nkx2.2 (1:50 for IF) were obtained from the Developmental Studies Hybridoma Bank (developed under the auspices of the NICHD and maintained by The University of Iowa, Department of Biology, Iowa City, IA 52242.Bank); biotinylated Lotus Tetragonolobus Lectin (LTL) (Vector laboratories; Cat \# B1325; 1:200). Secondary antibodies (all used at 1:500 for IF): Alexa 488 goat anti-rabbit (Invitrogen A-11034); Alexa 488 goat-anti mouse (Invitrogen A-11029); Alexa 555 goat anti-rabbit (Invitrogen A-21428), Alexa 594 goat anti-rabbit (Invitrogen A-11037); Alexa 594 goat anti-mouse (Invitrogen A-11032). Primers used in this study are presented in Supplementary Table 3. 


\section{Mouse skeletal preparations, in situ hybridization, scanning electron microscopy and histology}

Mouse skeletal preparations ${ }^{52}$, whole mount in situ hybridization ${ }^{53}$ and probes used in the $\operatorname{limb}^{54}$ have been described previously. Anti-sense RNA probes were prepared by transcribing linearized cDNA clones with SP6 and T7 polymerases using the digoxigenin labeling mix (Roche). Scanning electron microscopy methods and preparation of mouse tissues for histological analysis have been described previously ${ }^{55}$. For histological analysis, sections were stained with haematoxylin and eosin (H\&E), Elastica van Gieson (EvG) or Silver using standard procedures.

\section{Cell culture}

Control and mutant MEFs were isolated from trunks of $13.5 \mathrm{dpc}$ littermate embryos by standard methods ${ }^{56}$ and cultured as primary cell lines for up to 5 passages in Dulbecco's Modified Eagle Medium (DMEM, Invitrogen) containing 10\% fetal bovine serum (FBS). Mouse inner medullary collecting duct (IMCD3) cells were grown under the same conditions. Human dermal fibroblasts were derived from skin biopsies from ARPKD affected individual B155 and age- and sex-matched control individuals using standard techniques. Human fibroblasts and HEK293T cells were maintained in DMEM (Sigma, St. Louis, IL, USA) with $4500 \mathrm{mg} / \mathrm{L}$ glucose and 10\% FBS (HyClone, SH30071.03HI). RPE-1 cells and RPE-1 cells with stable expression of ARL13B-GFP were cultured in ATCC formulated DMEM:F12 Medium (Catalog No. 30-2006) with 10\% FBS and $0.01 \mathrm{mg} / \mathrm{ml}$ hygromycin B. Cultures of immortalized mouse kidney cells from heterozygous $P k d 1^{\text {flox } /-}$ and $P k d 2^{+/-}$mice as well as $P k d 1^{--}$and $P k d 2^{-/}$null mutant cells (derived by inactivating the flox allele with Cre recombinase via transfection in the case of $P k d 1^{57}$ and via spontaneous in-vivo conversion for $P k d 2^{58,59}$ ) (gifts of S. Somlo) were cultured in DMEM containing $2 \%$ fetal bovine serum (FBS), 10,000U/ $\mu \mathrm{l}$ IFN $\gamma, 10 \mathrm{mg} / \mathrm{L}$ Insulin, $6.7 \mu \mathrm{g} / \mathrm{L}$ Selenium, 5.5mg/L Transferrin and 1.2ug/L Triiodo-L-thyronine. Established cell lines, human fibroblasts and primary MEFs tested negative for mycoplasma.

\section{IF analysis of mouse and human cells and mouse tissue sections}

For IF analysis, cells were grown on glass coverslips to approximately $80 \%-90 \%$ confluency in DMEM + $10 \%$ FBS, at which time the medium was replaced with DMEM containing $0.2 \% \mathrm{FBS}$ for $24 \mathrm{~h}$ or $0.1 \% \mathrm{FBS}$ for $48 \mathrm{~h}$ to induce ciliogenesis. For analysis of SMO the reduced serum media was supplemented with SAG (100nM, Alexis Biochemicals) or diluent alone for $24 \mathrm{~h}$. For cell cycle analysis cells were synchronized by serum starvation as above for $24 \mathrm{~h}$, the media was replaced with DMEM $+10 \%$ FBS and cells were fixed at different timepoints to optimize capture of cells at different stages of the cell cycle. Cells were fixed in either ice-cold methanol for 5 minutes (co-localization experiments involving Abnova anti-DZIP1L and PC2 antibodies) or 4\% paraformaldehyde (PFA) for 10 minutes. PFA fixed cells were permeabilised in PBTX (PBS, 0.1\% Triton X-100) for 10 minutes. Fixed cells were washed in PBS, and incubated with $2 \%$ bovine serum albumin in PBS for 1 hour, followed by 1 hour with primary antibody. After brief washes in PBS, cells were incubated with secondary antibody for 1 hour, and 10 minutes wash with 6-Diamidino-2-Phenylindole (DAPI) to stain nuclei, and mounted on glass slides with Dako Fluorescence mounting 
medium and imaged on a Zeiss LSM-710 or Olympus FluoView upright laser scanning confocal microscope with a $63 \mathrm{x}$ or $100 \mathrm{x}$ objectives. For acquisition of 3D structured illumination superresolution microscopy (3D-SIM) images the sample preparation was modified to use high-precision coverslips $(\# 1.5 \mathrm{H})$ and VectaShield H-1000 mounting media (Vector Laboratories) in an effort to minimise spherical aberration. 3D-SIM was performed using a DeltaVision OMX v4 Blaze microscope (GE Healthcare) equipped with 488, 568 and $647 \mathrm{~nm}$ lasers, and BGR-FR filter drawer as previously described ${ }^{60}$.

IF of embryo sections was performed as previously described ${ }^{61}$. For quantification of cilia in limb sections, three confocal Z-stacks were performed in each of 3 different sections from the limb ( 9 stacks in total per limb). Given the density of cells in the tissue, and to attempt to account for orientation of cilia in different planes, nuclei were manually counted from the third image of each 5-image stack, while cilia were counted from all 5. Note that the final cilia/nuclei ratio does not necessarily represent the actual percentage of ciliated cells in the tissue, but is a relative value across the samples. The same conclusion was obtained from counting both nuclei and cilia from just the third image of each stack.

\section{Quantitative real time RT-PCR (qRT-PCR)}

MEFs were cultured in DMEM containing 10\% FBS to 80\% confluency, before replacing with DMEM+0.2\% FBS for 24h. For SAG treatment experiment, the reduced serum media was supplemented with SAG (100nM, Alexis Biochemicals) or diluent alone for 24h. Total RNA was extracted using TriReagent (Sigma-Aldrich), and first-strand cDNA synthesis was performed using SuperScript III First-Strand Synthesis system (Invitrogen) using oligo dT primers. cDNA was amplified with ABI SYBR Green PCR master mix using transcript specific primers (Supplementary Table 3). Temperature cycling and real-time detection of SYBR Green fluorescence used an Applied Biosystems 7900HT Fast Real-Time PCR system. Technical triplicate reactions were performed for each sample (gene of interest and normalising gene). Relative transcript levels were determined using the $2^{-\Delta \mathrm{CT}}$ method.

\section{Zebrafish strains and maintenance}

Zebrafish (Danio rerio) were raised under standard conditions at $28^{\circ} \mathrm{C}$. TU and $\mathrm{AB}$ wildtype embryo strains were used and staged according to Kimmel ${ }^{62}$. The $T g($ Wt $1 b:: G F P)$ strain has been described previously ${ }^{26}$.

\section{Zebrafish embryo microinjections}

Morpholinos were synthesized by Gene Tools and re-suspended in water at a stock concentration of $1 \mathrm{mM}$. Morpholinos were injected into one- to two-cell stage eggs, with each morpholino injected at least four times for each analysis. The following volumes of morpholinos were injected: $1.5 \mathrm{nl}$ of translation blocking morpholino $(0.25 \mathrm{mM}$ working solution), i1e 2 morpholino $(0.05 \mathrm{mM})$, i4e 5 morpholino $(0.35 \mathrm{mM})$ and $0.75 \mathrm{nl}$ e $5 \mathrm{i} 5$ morpholino $(1 \mathrm{mM})$. For rescue experiments, sense mRNAs of zebrafish and human DZIP1L (in the pSP64T or pCS2 vectors) were in vitro transcribed using the mMessage mMachine kit (Life technologies). For rescue of dzip11 morphants, embryos were co-injected with zebrafish dzip11 mRNA (100ng/ $\mu \mathrm{l})$. 


\section{RT-PCR and in situ hybridization with zebrafish embryos}

Zebrafish total RNA was isolated by Trizol (Ambion Life Technologies, \# 15596018). RTPCR was carried out using one-step RT-PCR kit (QIAGEN,\# 210212). $1 \mu \mathrm{g}$ of total RNA from each experimental group was used to synthesize the 1st strand cDNA. dzip11 specific primers were used to check mis-splicing induced by the morpholinos. actinb1 and efla RTPCR product levels were used as loading control. Whole mount in situ hybridization with the zebrafish dzip11, ptc1, eng1a and $n k \times 2.2 b$ antisense mRNA probes was performed according to standard protocols.

\section{Whole-mount IF staining of zebrafish embryos and confocal microscopy}

Zebrafish embryos were fixed in Dent's fixative (80\% methanol, 20\% DMSO) for 3 hours or $4 \%$ paraformaldehyde for 2 hours at room temperature, followed by storing in absolute methanol at $-20^{\circ} \mathrm{C}$. Embryos were then rehydrated in a decreasing methanol:PBS gradient. Blocking buffer (1\% (w/v) BSA, 1\% DMSO, 0.5\% Triton X-100 and 2\% sheep serum in PBS) was used to block the embryos for 1 hour. The embryos were then incubated with primary antibodies at $4{ }^{\circ} \mathrm{C}$ over-night. After washing embryos with PBDT (1\% (w/v) BSA, $1 \%$ DMSO, $0.5 \%$ Triton X-100 in PBS), the embryos were then incubated in fluorophoreconjugated secondary antibodies and DAPI for 3 hours at room temperature. The stained embryos were mounted in $70 \%$ glycerol and imaged by confocal microscopy. Image acquisition and analysis was performed using the Olympus FluoView FV10-ASW software and an Olympus FluoView upright confocal microscope.

\section{Generation of dzip1/CRISPR mutants}

Design of sgRNAs: sgRNAs for the dzip11 gene were designed using the web tool $\mathrm{CHOPCHOP}^{63}$. Target sites for the sgRNAs were designed by seeking sequences corresponding to the GGN18NGG sequence in the DNA. Two sgRNAs, targeting exon 2 and exon 13, respectively, were designed. To check off-target effects of the sgRNAs, basic local alignment search tool (BLAST) was used and the results were visually analysed. Off-target sequences with no mismatches in the last $15 \mathrm{nt}$, including the NGG PAM, were discarded. Synthesis of sgRNAs: sgRNAs were synthesized based on a modified two-component system described by Bassett and colleagues ${ }^{64}$. Briefly, PCR was performed with a forward oligonucleotide encoding the T7 polymerase binding site and the sgRNA target sequence and a common reverse oligonucleotide encoding the remainder of the sgRNA sequence using Phusion polymerase (NEB, M0530S) (see Supplementary Table 3 for the oligonucleotide sequences). In vitro transcription was performed using 250ng of the purified DNA template with MEGAshortscript T7 kit (Ambion, AM1354M) and the sgRNAs were purified as per manufacturer's instruction. Micro-injection of zebrafish eggs: A mixture of $800 \mathrm{ng}$ of the Cas9 protein (Toolgen) and 500ng of each sgRNA were incubated at $37^{\circ} \mathrm{C}$ for 15 minutes. One-cell stage embryos were injected with $1 \mathrm{nl}$ of the mixture. Genomic DNA extraction and PCR analysis to identify mutations: Genomic DNA extraction from embryos and fins from adult fish were done using the alkaline lysis method ${ }^{65}$. For embryonic genomic DNA extraction, 8-10 single embryos at 2 dpf were used. To identify the mutations, primers were designed to bind upstream and downstream of the expected doublestranded breaks in exon 2 and exon 13 (Supplementary Table 3). The PCR amplified 
genomic DNA region from the mutants were TOPO-cloned and sequenced to confirm the deletion.

\section{Yeast two hybrid screen, immunoprecipitation and western blot}

Yeast two hybrid screen with human DZIP1L protein (three fragments: aa 1-232, 200-540 and 540-767) was performed by Myriad pharmaceuticals. For immunoprecipitation of endogenous DZIP1L and SEPT2 and vice versa, RPE-1 or HEK293T cells were lysed in $1 \mathrm{ml}$ of cell lysis buffer (20mM Tris at PH 8.0, 137mM NaCl, $1 \%$ Triton X-100, 2mM EDTA in PBS) with proteinase inhibitor cocktail (Roche, Switzerland). Protein concentrations were estimated by using Bio-Rad protein assay reagent (BioRad Laboratories, USA) according to the manufacturer's instructions and reading the absorbance at $595 \mathrm{~nm}$ with a spectrophotometer. Equal amounts of total protein lysates $(0.5 \mathrm{mg}-10 \mathrm{mg})$ were incubated with DZIP1L or SEPT2 antibodies $(2 \mu \mathrm{g})$ and protein A-agarose (Oncogene Research Products, USA) overnight at $4^{\circ} \mathrm{C}$. The immunoprecipitates were washed 5 times with cell lysis buffer, boiled in Laemmili buffer for $5 \mathrm{~min}$ and resolved by SDS-PAGE. For western blot analysis, cells were lysed in RIPA buffer (50mM Tris-HCL, pH7.2, 150mM NaCl, $1 \%$ Triton $\mathrm{x}-100,1 \%(\mathrm{w} / \mathrm{v})$ deoxycholic acid, $0.1 \%(\mathrm{w} / \mathrm{v})$ sodium dodecyl sulphate, $0.25 \mathrm{mM}$ EDTA with proteinase inhibitor cocktail). The proteins in the lysate were separated by SDSPAGE. For interaction of SEPT2, different DZIP1L constructs and NPHP1, MKS1, B9D1 and CEP89, transient transfections into HEK293T cells were carried out using JetPrimePolyPlus transfection kit (PolyPlus Transfections) according to the manufacturer's instructions. Cell lysates were prepared in lysis buffer (20mM Tris-HCl, pH7.4, 150mM $\mathrm{NaCl}, 1 \mathrm{mM}$ EDTA, $0.25 \%$ deoxycholate, $1 \% \mathrm{NP}-40$ and protease inhibitors). ImmunoCruz IP/WB Optima C beads (Santa cruz Biotechnology INC) were incubated with $2 \mu \mathrm{g}$ of M2 FLAG antibody (F1804; Sigma) for 3 hours at $4^{\circ} \mathrm{C}$ and subsequently washed in lysis buffer. Control and transiently transfected cell lysates were added to the antibody-beads conjugate, and incubated further overnight for $4^{\circ} \mathrm{C}$. Immunoprecipitates were washed in lysis buffer, separated by SDS PAGE, transferred onto PVDF membranes and probed with rabbit antiHA antibodies (77-5500; Thermo Fisher Scientific) followed by goat-anti rabbit-HRP secondary antibody (Biorad) and chemiluminescence (Super Signal West Pico; Thermo Fisher Scientific).

\section{Alignment of DZIP1L amino-acid sequences}

Human, mouse and zebrafish DZIP1L sequence alignment was performed using the MegAlign software (DNASTAR Lasergene 12 Core Suite) following the Clustal W Method.

\section{Statistical analyses}

Statistical analyses used Prism version 4.0, 6.0 or 7.0 software (Graphpad). In most cases Pvalues are based on an unpaired Student's $t$-test (see figure legends for details), the mean value is shown and error bars represent standard error of the mean (SEM). In these cases variances are not significantly different. The exception is the analysis in Fig. $3 \mathrm{~m}$ where both the sample sizes and variances are unequal, and an unpaired $t$-test was used with Welch's correction. The significance in this case was confirmed by a Student's $t$-test on log transformed data where the variances are equalized (not shown). For animal experiments no specific randomization or routine blinding strategies were employed. Regardless of whether 
statistical tests were applied, as per standard practice, all animal experiments were performed on multiple embryos/mice/morphants or primary cell lines derived from multiple independent embryos. For both mice and zebrafish the number of individuals used in each experiment is detailed in the text and/or relevant figure legend.

\section{Supplementary Material}

Refer to Web version on PubMed Central for supplementary material.

\section{Acknowledgments}

The authors would like to thank the patients and their families for their cooperation and interest in the study, M. T. Toh and Y. T. Koh for technical assistance, W. I. Goh of the Institute of Medical Biology's Microscopy Unit for help with superresolution microscopy, J. Lefevre and N. Hamilton for advice on statistical analyses, and C. Cortés, M. Pitt, F. Olsson, L. Zhao, L. Wilkinson and P. Karaith Oliva for assistance, insightful discussion and advice. Thanks also to C. Has for kindly providing control fibroblasts, S. Somlo, M. Ma and K. Dong for Pkd1 and Pkd2 mutant cells, L. Lei for the RPE-1 cell line stably expressing Arl13b-GFP, L. Pelletier for basal body and transition zone protein cDNA clones, and R. Witzgall, G. Wu and R. Rohatgi for antibodies. We would also like to thank staff of the University of Queensland (UQ) QBP animal house for help with mouse husbandry, and the Australian Phenomics Facility for maintaining mice throughout the screen. Confocal microscopy at UQ was carried out in the Institute for Molecular Bioscience Dynamic Imaging Facility for Cancer Biology, developed with the generous support of the Australian Cancer Research Foundation. We also acknowledge the Australian Microscopy \& Microanalysis Facility (AMMRF) at the Centre for Microscopy and Microanalysis at UQ. MH, SN, VF and CB are employees of Bioscientia/Sonic Healthcare, CW was a recipient of a University of Queensland Vice-Chancellor's Senior Research Fellowship and SR is a Senior Principal Investigator with the Institute of Molecular and Cell Biology, Singapore. MHL is a Senior Principal Research Fellow of the Australian National Health and Medical Research Council (NHMRC). FH is the Warren E. Grupe Professor. This work was supported by grants from the German Research Fund (DFG) to KZ and CB, the DFG Collaborative Research Centre (SFB) KIDGEM 1140 and the Federal Ministry of Education and Research (BMBF, 01GM1515C, project 2.3) to CB, the Australian NHMRC to CW (APP1045464), the National Institutes of Health NIH (DK068306) to FH and the Agency for Science, Technology and Research (A* STAR) of Singapore to WH and SR. This paper is dedicated to the memory of Markus Nauck, a friend and colleague, who recently passed away.

\section{References}

1. Guay-Woodford LM, et al. Consensus expert recommendations for the diagnosis and management of autosomal recessive polycystic kidney disease: report of an international conference. J Pediatr. 2014; 165:611-7. [PubMed: 25015577]

2. Bergmann C, et al. Clinical consequences of PKHD1 mutations in 164 patients with autosomalrecessive polycystic kidney disease (ARPKD). Kidney Int. 2005; 67:829-48. [PubMed: 15698423]

3. Habbig S, Liebau MC. Ciliopathies - from rare inherited cystic kidney diseases to basic cellular function. Mol Cell Pediatr. 2015; 2:8. [PubMed: 26542298]

4. Garcia-Gonzalo FR, et al. A transition zone complex regulates mammalian ciliogenesis and ciliary membrane composition. Nat Genet. 2011; 43:776-84. [PubMed: 21725307]

5. Jensen VL, et al. Formation of the transition zone by Mks5/Rpgrip1L establishes a ciliary zone of exclusion (CIZE) that compartmentalises ciliary signalling proteins and controls PIP2 ciliary abundance. EMBO J. 2015; 34:2537-56. [PubMed: 26392567]

6. Reiter JF, Blacque OE, Leroux MR. The base of the cilium: roles for transition fibres and the transition zone in ciliary formation, maintenance and compartmentalization. EMBO Rep. 2012; 13:608-18. [PubMed: 22653444]

7. Guay-Woodford LM. Autosomal recessive polycystic kidney disease: the prototype of the hepatorenal fibrocystic diseases. J Pediatr Genet. 2014; 3:89-101. [PubMed: 25632369]

8. Wang S, Luo Y, Wilson PD, Witman GB, Zhou J. The autosomal recessive polycystic kidney disease protein is localized to primary cilia, with concentration in the basal body area. J Am Soc Nephrol. 2004; 15:592-602. [PubMed: 14978161] 
9. Zhang MZ, et al. PKHD1 protein encoded by the gene for autosomal recessive polycystic kidney disease associates with basal bodies and primary cilia in renal epithelial cells. Proc Natl Acad Sci U S A. 2004; 101:2311-6. [PubMed: 14983006]

10. Wu Y, et al. Kinesin-2 mediates physical and functional interactions between polycystin-2 and fibrocystin. Human molecular genetics. 2006; 15:3280-3292. [PubMed: 17008358]

11. Wang S, et al. Fibrocystin/polyductin, found in the same protein complex with polycystin-2, regulates calcium responses in kidney epithelia. Mol Cell Biol. 2007; 27:3241-52. [PubMed: 17283055]

12. Kim I, et al. Fibrocystin/polyductin modulates renal tubular formation by regulating polycystin-2 expression and function. J Am Soc Nephrol. 2008; 19:455-68. [PubMed: 18235088]

13. Delling M, DeCaen PG, Doerner JF, Febvay S, Clapham DE. Primary cilia are specialized calcium signalling organelles. Nature. 2013; 504:311-4. [PubMed: 24336288]

14. DeCaen PG, Delling M, Vien TN, Clapham DE. Direct recording and molecular identification of the calcium channel of primary cilia. Nature. 2013; 504:315-8. [PubMed: 24336289]

15. Delling M, et al. Primary cilia are not calcium-responsive mechanosensors. Nature. 2016; 531:656-60. [PubMed: 27007841]

16. Eisenberger T, et al. An efficient and comprehensive strategy for genetic diagnostics of polycystic kidney disease. PLoS One. 2015; 10:e0116680. [PubMed: 25646624]

17. Glazer AM, et al. The $\mathrm{Zn}$ finger protein Iguana impacts Hedgehog signaling by promoting ciliogenesis. Dev Biol. 2010; 337:148-56. [PubMed: 19852954]

18. Tay SY, et al. The iguana/DZIP1 protein is a novel component of the ciliogenic pathway essential for axonemal biogenesis. Dev Dyn. 2010; 239:527-34. [PubMed: 20014402]

19. Wolff $\mathrm{C}$, et al. iguana encodes a novel zinc-finger protein with coiled-coil domains essential for Hedgehog signal transduction in the zebrafish embryo. Genes Dev. 2004; 18:1565-76. [PubMed: 15198976]

20. Sekimizu K, et al. The zebrafish iguana locus encodes Dzip1, a novel zinc-finger protein required for proper regulation of Hedgehog signaling. Development. 2004; 131:2521-32. [PubMed: 15115751]

21. Kim HR, Richardson J, van Eeden F, Ingham PW. Gli2a protein localization reveals a role for Iguana/DZIP1 in primary ciliogenesis and a dependence of Hedgehog signal transduction on primary cilia in the zebrafish. BMC Biol. 2010; 8:65. [PubMed: 20487519]

22. Wang C, Low WC, Liu A, Wang B. Centrosomal protein DZIP1 regulates Hedgehog signaling by promoting cytoplasmic retention of transcription factor GLI3 and affecting ciliogenesis. J Biol Chem. 2013; 288:29518-29. [PubMed: 23955340]

23. Papathanasiou $\mathrm{P}$, et al. A recessive screen for genes regulating hematopoietic stem cells. Blood. 2010; 116:5849-58. [PubMed: 20610815]

24. Huangfu D, Anderson KV. Cilia and Hedgehog responsiveness in the mouse. Proc Natl Acad Sci U S A. 2005; 102:11325-30. [PubMed: 16061793]

25. Litingtung Y, Dahn RD, Li Y, Fallon JF, Chiang C. Shh and Gli3 are dispensable for limb skeleton formation but regulate digit number and identity. Nature. 2002; 418:979-83. [PubMed: 12198547]

26. Perner B, Englert C, Bollig F. The Wilms tumor genes wt1a and wt1b control different steps during formation of the zebrafish pronephros. Dev Biol. 2007; 309:87-96. [PubMed: 17651719]

27. Kobayashi T, Dynlacht BD. Regulating the transition from centriole to basal body. J Cell Biol. 2011; 193:435-44. [PubMed: 21536747]

28. Hu Q, et al. A septin diffusion barrier at the base of the primary cilium maintains ciliary membrane protein distribution. Science. 2010; 329:436-9. [PubMed: 20558667]

29. Chih B, et al. A ciliopathy complex at the transition zone protects the cilia as a privileged membrane domain. Nat Cell Biol. 2012; 14:61-72.

30. Ong AC, et al. Polycystin-1 expression in PKD1, early-onset PKD1, and TSC2/PKD1 cystic tissue. Kidney Int. 1999; 56:1324-33. [PubMed: 10504485]

31. Bergmann C, et al. Spectrum of mutations in the gene for autosomal recessive polycystic kidney disease (ARPKD/PKHD1). J Am Soc Nephrol. 2003; 14:76-89. [PubMed: 12506140] 
32. Bergmann C, et al. PKHD1 mutations in autosomal recessive polycystic kidney disease (ARPKD). Hum Mutat. 2004; 23:453-63. [PubMed: 15108277]

33. Gunay-Aygun M, et al. Correlation of kidney function, volume and imaging findings, and PKHD1 mutations in 73 patients with autosomal recessive polycystic kidney disease. Clin J Am Soc Nephrol. 2010; 5:972-84. [PubMed: 20413436]

34. Nakanishi K, Sweeney WE Jr, Zerres K, Guay-Woodford LM, Avner ED. Proximal tubular cysts in fetal human autosomal recessive polycystic kidney disease. J Am Soc Nephrol. 2000; 11:760-3. [PubMed: 10752536]

35. Williams SS, Cobo-Stark P, James LR, Somlo S, Igarashi P. Kidney cysts, pancreatic cysts, and biliary disease in a mouse model of autosomal recessive polycystic kidney disease. Pediatr Nephrol. 2008; 23:733-41. [PubMed: 18286309]

36. Bakeberg JL, et al. Epitope-tagged Pkhd1 tracks the processing, secretion, and localization of fibrocystin. J Am Soc Nephrol. 2011; 22:2266-77. [PubMed: 22021705]

37. Woollard JR, et al. A mouse model of autosomal recessive polycystic kidney disease with biliary duct and proximal tubule dilatation. Kidney international. 2007; 72:328-336. [PubMed: 17519956]

38. Davis EE, Katsanis N. The ciliopathies: a transitional model into systems biology of human genetic disease. Curr Opin Genet Dev. 2012; 22:290-303. [PubMed: 22632799]

39. Boulter C, et al. Cardiovascular, skeletal, and renal defects in mice with a targeted disruption of the Pkd1 gene. Proceedings of the National Academy of Sciences of the United States of America. 2001; 98:12174-12179. [PubMed: 11593033]

40. Kolpakova-Hart E, et al. Growth of cranial synchondroses and sutures requires polycystin-1. Dev Biol. 2008; 321:407-19. [PubMed: 18652813]

41. Rossi A, et al. Genetic compensation induced by deleterious mutations but not gene knockdowns. Nature. 2015; 524:230-3. [PubMed: 26168398]

42. Blum M, De Robertis EM, Wallingford JB, Niehrs C. Morpholinos: Antisense and Sensibility. Dev Cell. 2015; 35:145-9. [PubMed: 26506304]

43. Zhang B, et al. GSK3beta-Dzip1-Rab8 cascade regulates ciliogenesis after mitosis. PLoS Biol. 2015; 13:e1002129. [PubMed: 25860027]

44. Diener DR, Lupetti P, Rosenbaum JL. Proteomic analysis of isolated ciliary transition zones reveals the presence of ESCRT proteins. Curr Biol. 2015; 25:379-84. [PubMed: 25578910]

45. Toriyama M, et al. The ciliopathy-associated CPLANE proteins direct basal body recruitment of intraflagellar transport machinery. Nat Genet. 2016; 48:648-56. [PubMed: 27158779]

46. Ma M, Tian X, Igarashi P, Pazour GJ, Somlo S. Loss of cilia suppresses cyst growth in genetic models of autosomal dominant polycystic kidney disease. Nat Genet. 2013; 45:1004-12. [PubMed: 23892607]

47. Braun DA, et al. Mutations in nuclear pore genes NUP93, NUP205 and XPO5 cause steroidresistant nephrotic syndrome. Nat Genet. 2016; 48:457-65. [PubMed: 26878725]

48. Zhou W, et al. FAN1 mutations cause karyomegalic interstitial nephritis, linking chronic kidney failure to defective DNA damage repair. Nat Genet. 2012; 44:910-5. [PubMed: 22772369]

49. Hoff S, et al. ANKS6 is a central component of a nephronophthisis module linking NEK8 to INVS and NPHP3. Nat Genet. 2013; 45:951-6. [PubMed: 23793029]

50. Andrews TD, et al. Massively parallel sequencing of the mouse exome to accurately identify rare, induced mutations: an immediate source for thousands of new mouse models. Open Biol. 2012; 2:120061. [PubMed: 22724066]

51. Rohatgi R, Milenkovic L, Scott MP. Patched1 regulates hedgehog signaling at the primary cilium. Science. 2007; 317:372-6. [PubMed: 17641202]

52. Bruce SJ, et al. Inactivation of Patched1 in the mouse limb has novel inhibitory effects on the chondrogenic program. J Biol Chem. 2010; 285:27967-81. [PubMed: 20576618]

53. Fowles LF, et al. Genomic screen for genes involved in mammalian craniofacial development. Genesis. 2003; 35:73-87. [PubMed: 12533789]

54. Butterfield NC, et al. Patched 1 is a crucial determinant of asymmetry and digit number in the vertebrate limb. Development. 2009; 136:3515-24. [PubMed: 19783740] 
55. Ashe A, et al. Mutations in mouse Ift144 model the craniofacial, limb and rib defects in skeletal ciliopathies. Hum Mol Genet. 2012; 21:1808-23. [PubMed: 22228095]

56. Todaro GJ, Green H. Quantitative studies of the growth of mouse embryo cells in culture and their development into established lines. J Cell Biol. 1963; 17:299-313. [PubMed: 13985244]

57. Shibazaki S, et al. Cyst formation and activation of the extracellular regulated kinase pathway after kidney specific inactivation of Pkd1. Hum Mol Genet. 2008; 17:1505-16. [PubMed: 18263604]

58. Grimm DH, et al. Polycystin-1 distribution is modulated by polycystin-2 expression in mammalian cells. J Biol Chem. 2003; 278:36786-93. [PubMed: 12840011]

59. Wu G, et al. Somatic inactivation of Pkd2 results in polycystic kidney disease. Cell. 1998; 93:17788. [PubMed: 9568711]

60. Xie W, Horn HF, Wright GD. Superresolution Microscopy of the Nuclear Envelope and Associated Proteins. Methods Mol Biol. 2016; 1411:83-97. [PubMed: 27147035]

61. Metzis V, et al. Patched1 is required in neural crest cells for the prevention of orofacial clefts. Hum Mol Genet. 2013; 22:5026-35. [PubMed: 23900075]

62. Kimmel CB, Ballard WW, Kimmel SR, Ullmann B, Schilling TF. Stages of embryonic development of the zebrafish. Dev Dyn. 1995; 203:253-310. [PubMed: 8589427]

63. Montague TG, Cruz JM, Gagnon JA, Church GM, Valen E. CHOPCHOP: a CRISPR/Cas9 and TALEN web tool for genome editing. Nucleic Acids Res. 2014; 42:W401-7. [PubMed: 24861617]

64. Bassett AR, Tibbit C, Ponting CP, Liu JL. Highly efficient targeted mutagenesis of Drosophila with the CRISPR/Cas9 system. Cell Rep. 2013; 4:220-8. [PubMed: 23827738]

65. Yu C, Zhang Y, Yao S, Wei Y. A PCR based protocol for detecting indel mutations induced by TALENs and CRISPR/Cas9 in zebrafish. PLoS One. 2014; 9:e98282. [PubMed: 24901507] 

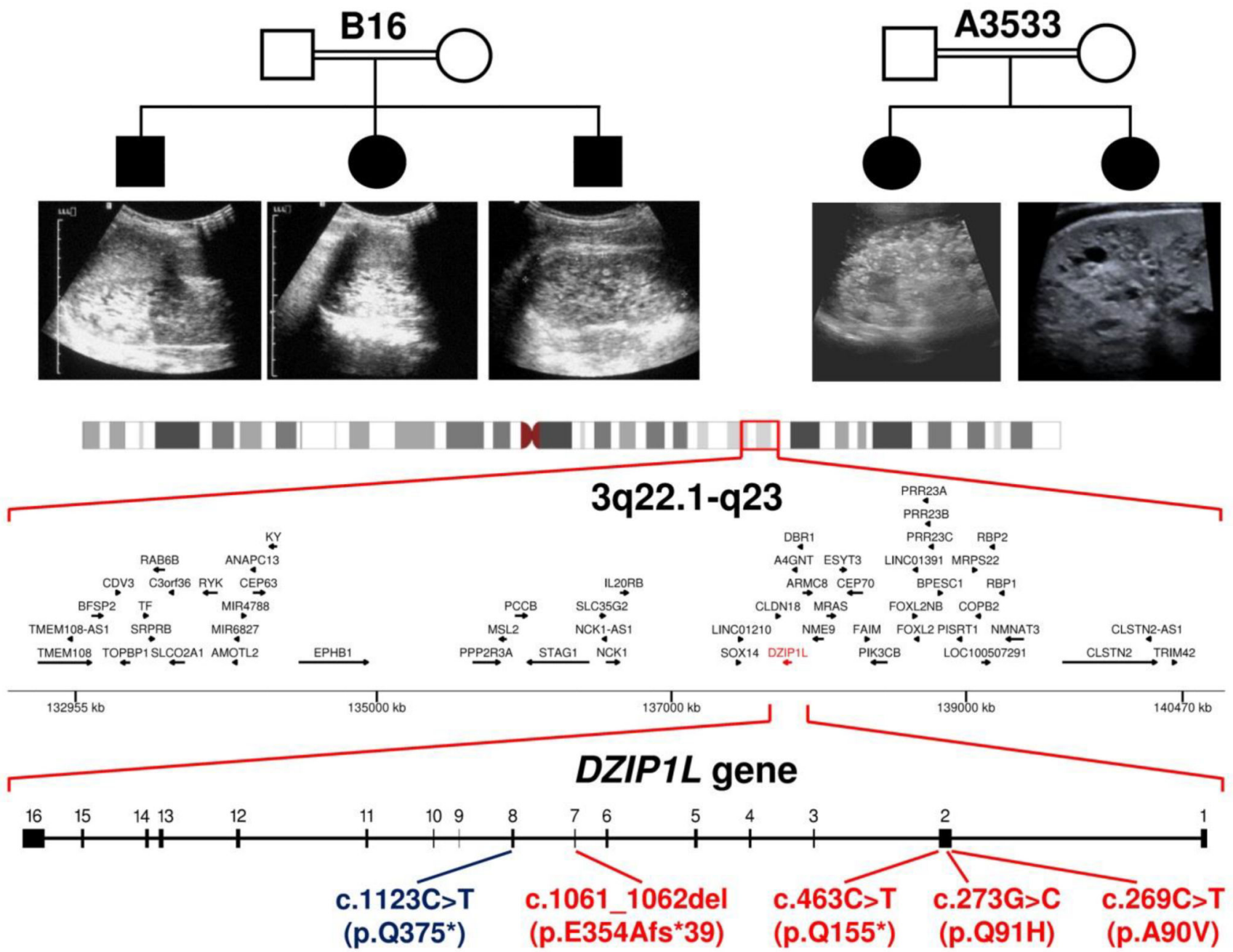

Figure 1.

Mapping of a new ARPKD locus on 3q22.1-q23 and identification of DZIP1L mutations.

Genome-wide SNP analysis performed in two unrelated consanguineous multiplex pedigrees (shown at top) with a total of five children affected by ARPKD, resulted in identification of a single overlapping $7.5 \mathrm{Mb}$ region of homozygosity on chromosome 3q22.1-q23. By various sequencing approaches, we identified different homozygous DZIP1L mutations in these and other consanguineous families with ARPKD (see text for details). In parallel, we identified an ENU-induced recessive mouse model (see Fig. 2) bearing the homozygous Dzip11 nonsense mutation c.1123C >T (p.Gln375*) (in blue). 

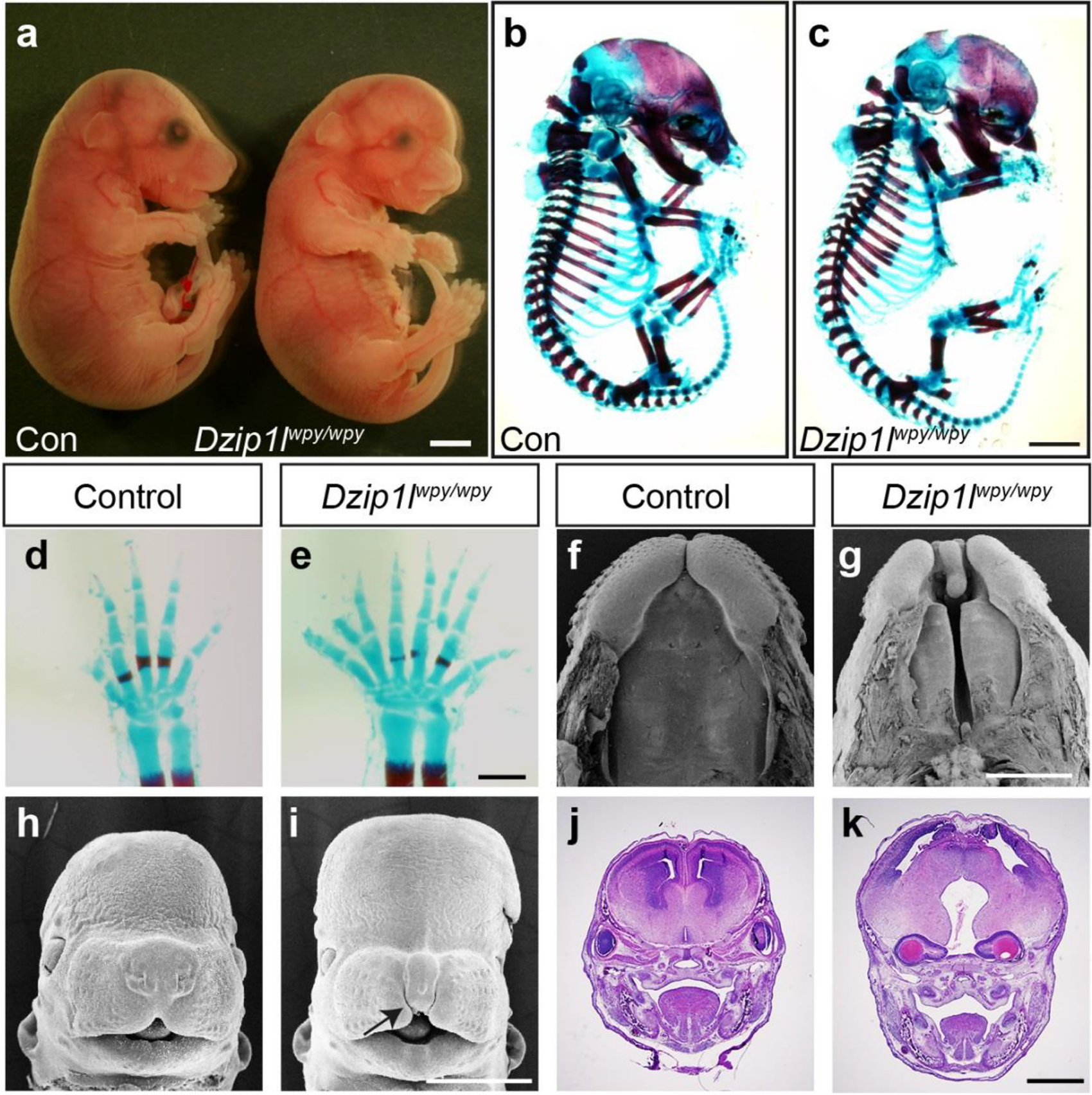

Figure 2.

Dzip1 1 wpy/wpy mutant embryos show multiple dysmorphic features. (a) Control (left) and mutant (right) embryos at $17.5 \mathrm{dpc}$. (b-e) Skeletal preps at 17.5dpc highlight defects in the

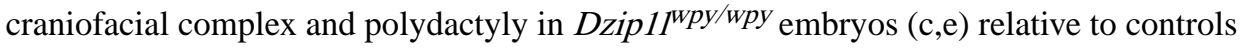
(b,d). Dzip1 1 wpy/wpy mutant embryos consistently show some degree of cleft palate (f,g; arrowhead in g) and bilateral cleft lip (f-i; arrows in g,i). Scanning electron micrographs of 15.5dpc embryos shown with lower jaw removed in f,g; $13.5 \mathrm{dpc}$ embryo heads in h,i. (j,k) In a subset of embryos ( $n=30 / 45$ scored) the eyes appeared abnormal, and in severe cases were buried within the skull (arrow in k). Images shown are of frontal sections through 15.5dpc 
embryonic heads. Note control embryos shown in $(b, f, j)$ are heterozygous embryos that show no obvious phenotypic differences from wild-type controls, as shown in (a,d,h). The limbs shown in (d,e) are from different embryos than those shown in $(b, c)$. Scale bars in $\mathrm{a}, \mathrm{b}, \mathrm{c}=2 \mathrm{~mm} ; \mathrm{d}, \mathrm{e}=0.5 \mathrm{~mm} ; \mathrm{f}-\mathrm{k}=1 \mathrm{~mm}$. All data presented are from embryos on a C57BL/ 6:C3H mixed background. 

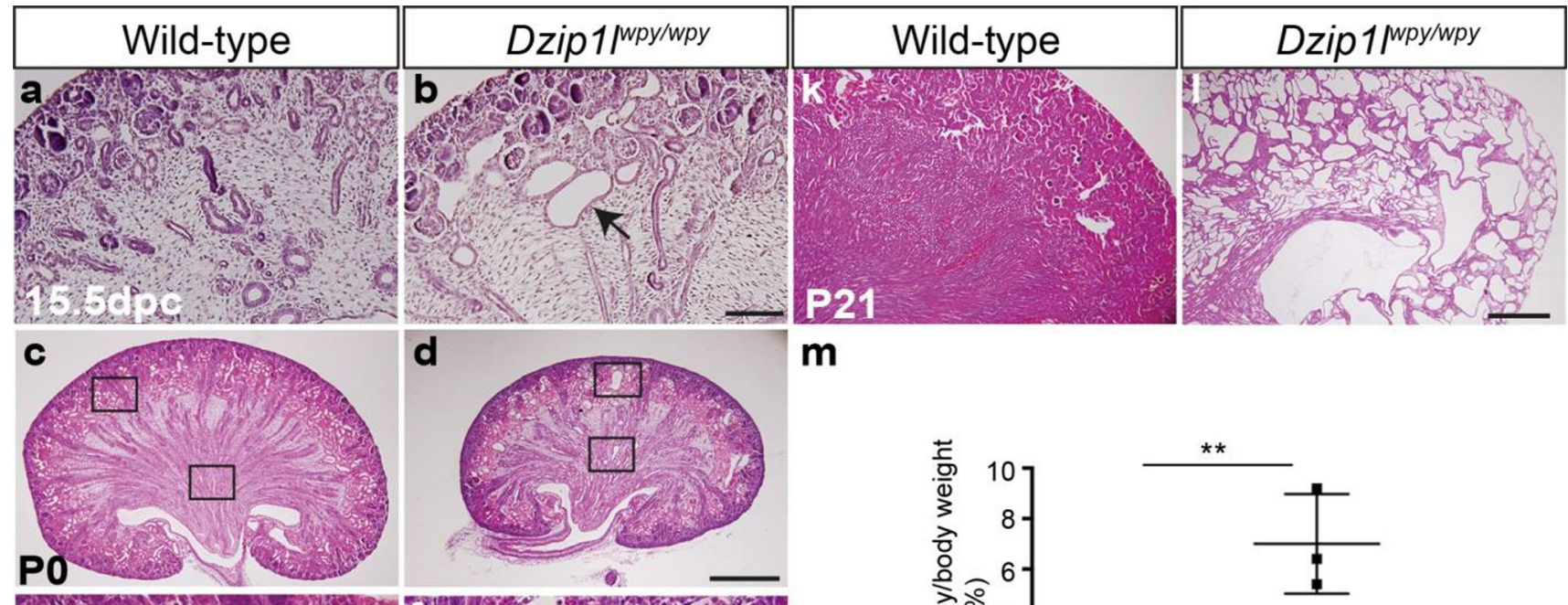

m
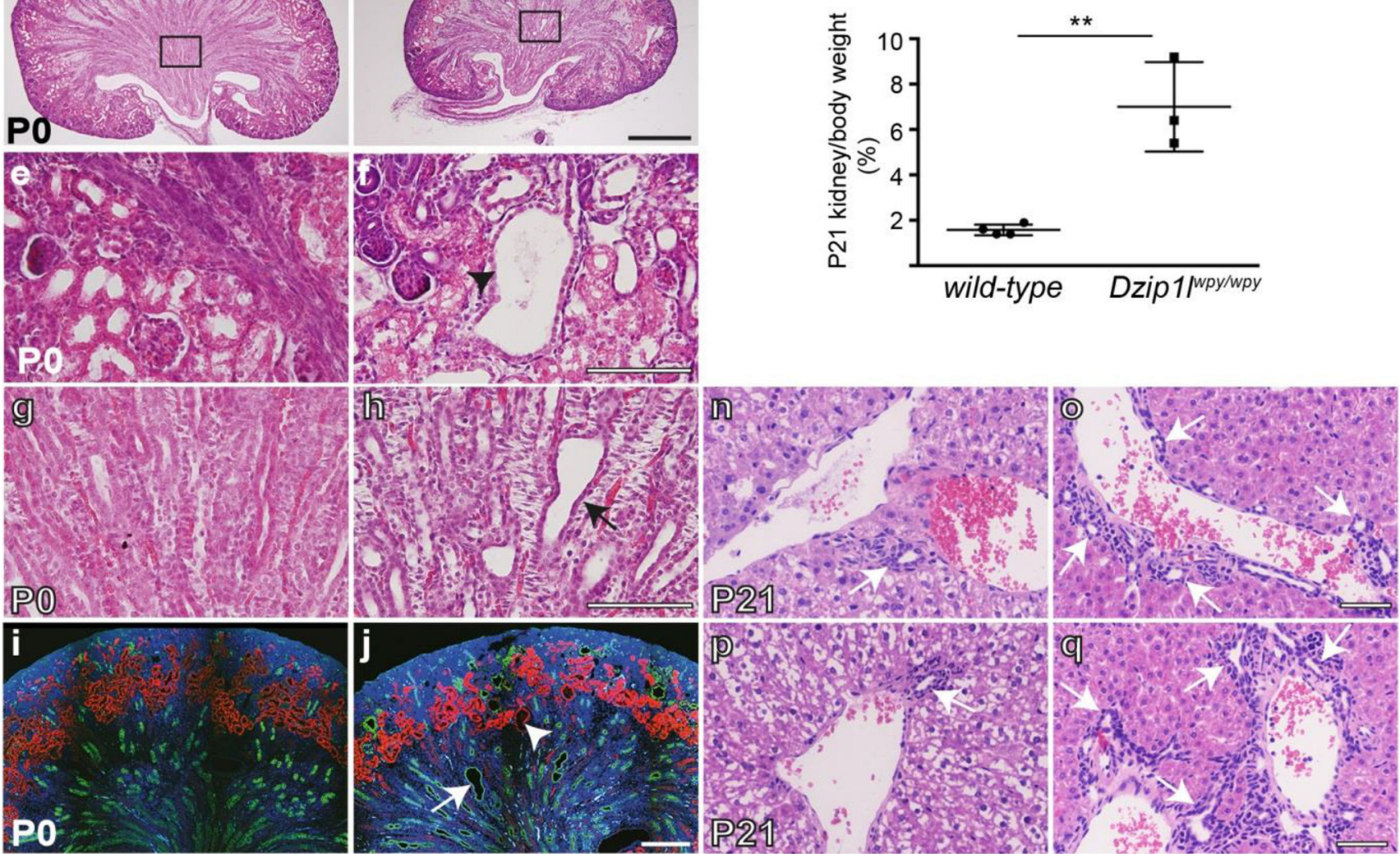

Figure 3.

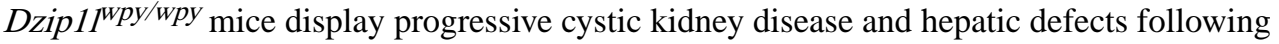
at least 4 crosses to a CD1 background. (a,b) Following H\&E staining, cortical cysts were evident from 15.5dpc in Dzip1 1 ${ }^{\text {wpy/wpy }}$ kidneys (arrow in b; $\mathrm{n}=4$ independent embryos). $(\mathrm{c}, \mathrm{d}, \mathrm{g}, \mathrm{h})$ At P0, both cortical cysts (arrowhead in $\mathrm{d}, \mathrm{g}$ ) and medullary dilatations (arrow in $d, h)$ were observed $(n=6)$. $(g, h)$ Show high power images of the regions marked in (d). $(e, f, i, j)$ Immunofluorescence analysis of sections from P0 kidneys stained with Aqp2 (collecting ducts; green), and LTL-biotin (proximal tubules; magenta). (i,j) Show high power images of the proximal tubule cyst (i) and collecting duct dilatation (j) marked with an arrowhead and arrow respectively in (f). (k,l) By P21, cysts extend throughout the kidney, and (m) when corrected for body weight Dzip1 $11^{\text {Wpy/wpy }}$ kidneys are heavier than wild-type ( $\mathrm{n}=3$ mutant, 4 wild-type embryos). Statistical analysis based on unpaired $t$-test with Welch's correction to account for unequal variances, ${ }^{*} \mathrm{p}=0.05$. Error bars show SEM. A 
significant difference was confirmed with a Student's $t$-test on log transformed data, where the variances are equalized (not shown). (n-q) H\&E staining of P21 liver sections. Arrows mark an excess of bile ducts, which nearly circumferentially surround portal vein branches in P21 Dzip11 ${ }^{\text {wpy/wPy }}$ mutant $(\mathrm{o}, \mathrm{q})$ compared to wild-type $(\mathrm{n}, \mathrm{p})$ mice, indicating DPM (n=2). Scale bars in a,b $=100 \mu \mathrm{m} ; \mathrm{c}-\mathrm{f}=200 \mu \mathrm{m}, \mathrm{k}, \mathrm{l}=500 \mu \mathrm{m}$ and $\mathrm{g}-\mathrm{j}$ and $\mathrm{n}-\mathrm{q}=50 \mu \mathrm{m}$. 

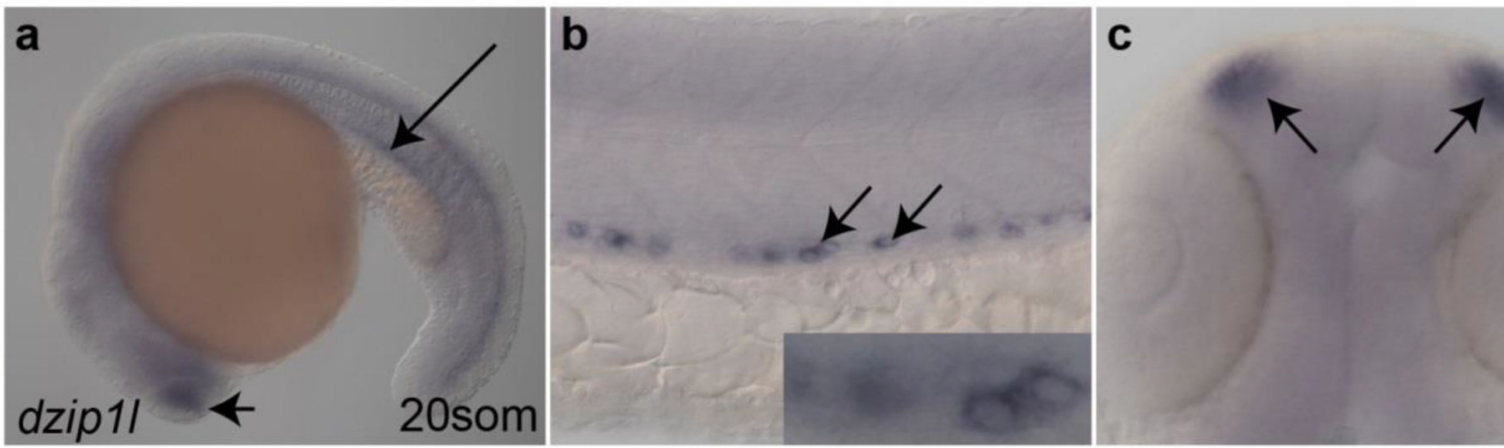

d

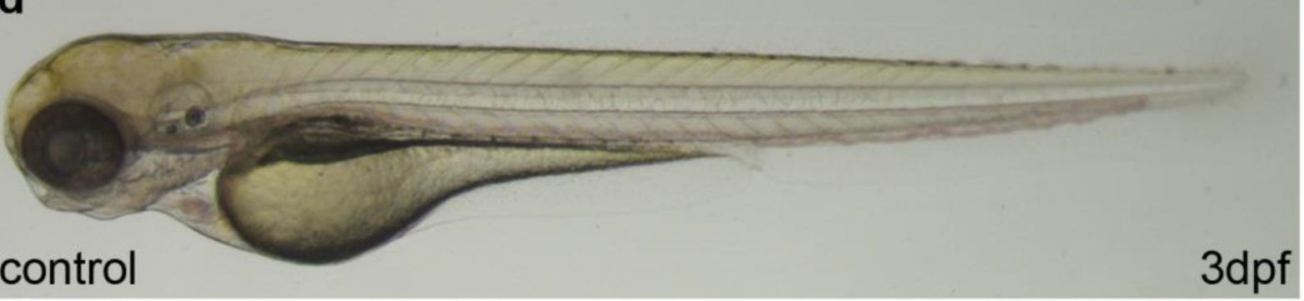

e control

f
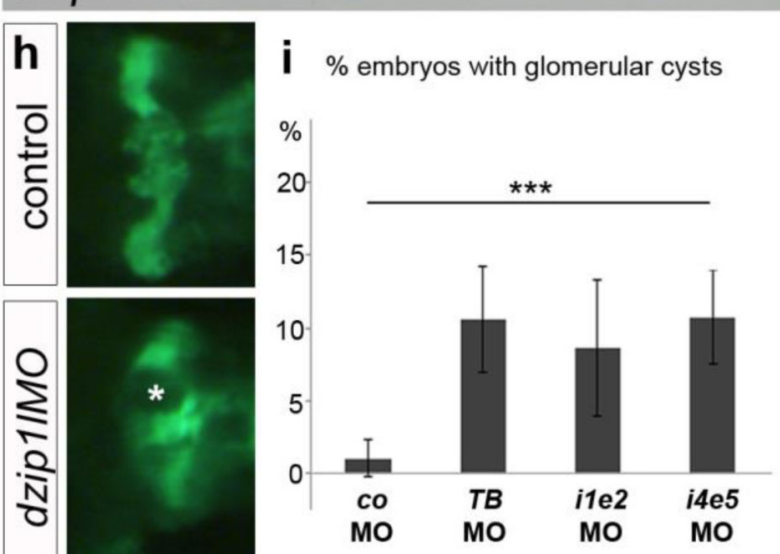

$3 \mathrm{dpf}$
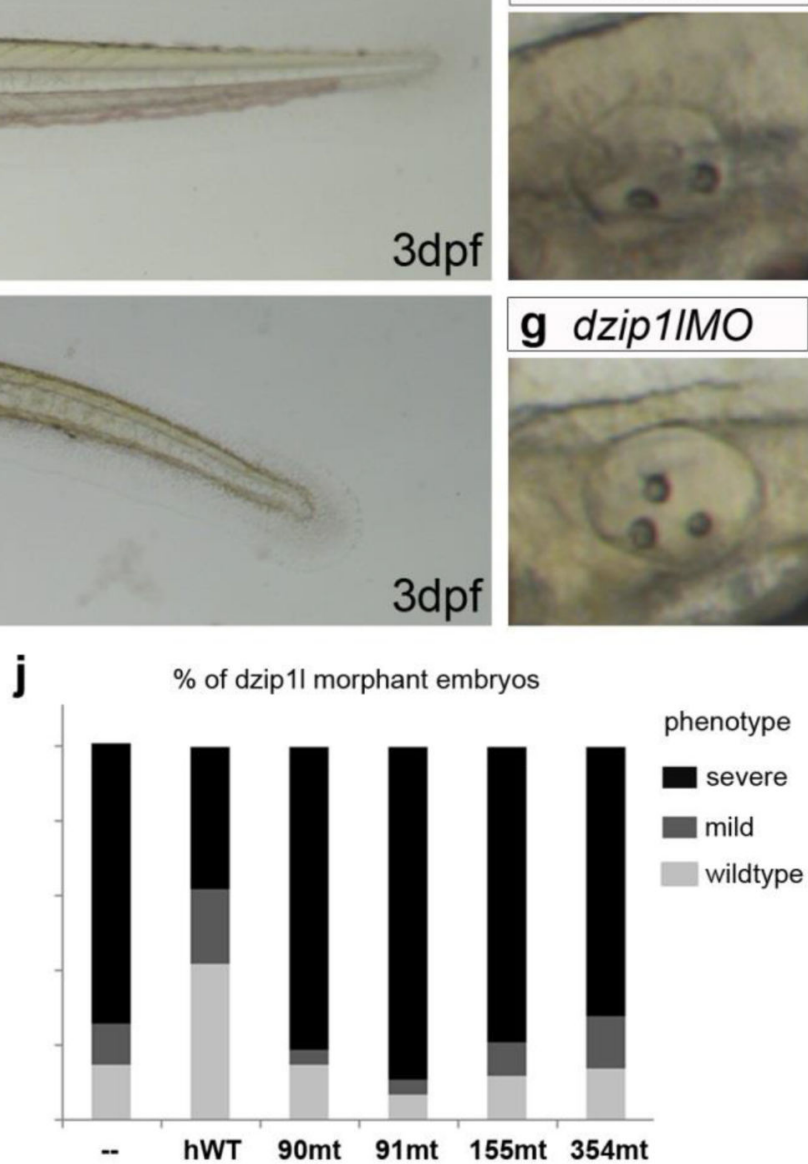

Figure 4.

DZIP1L localization overlaps with basal body, centrosome and transition zone markers. $(a, b)$ IF staining for DZIP1L (green) and acetylated-a-tubulin and $\boldsymbol{\gamma}$-tubulin (both in magenta) in serum starved IMCD3 kidney cells. DZIP1L staining overlaps with the basal body in ciliated cells (a) and the centrosome in non-ciliated cells (b). (c-d) Co-localization of DZIP1L (magenta) with the distal (CEP164, green in c) and subdistal (ODF2, green in d) appendage proteins. (e-i) DZIP1L (green) tracks with the centrioles (magenta) throughout all stages of the cell cycle in IMCD3 cells. (j) TCTN1 (magenta) and DZIP1L (cyan) co-localize at the transition zone in human dermal fibroblasts. ARL13B-GFP was used to mark the ciliary 
membrane around the axoneme (green in j). (k) 3D-SIM superresolution microscopy on RPE-1 (human retinal pigment epithelial) cells confirms both DZIP1L (magenta) and TCTN1 (cyan) are closely associated at the transition zone. Cilium labeled with ARL13BGFP (green). Nuclei are stained with DAPI (blue). Scale bars in $\mathrm{a}-\mathrm{I}=5 \mu \mathrm{m} ; \mathrm{j}=1 \mu \mathrm{m} ; \mathrm{k}=500$ $\mathrm{nm}$. DZIP1L stained with Sigma C-terminal antibody, except in panels c,d,j,k where the Abnova antibody was used. 

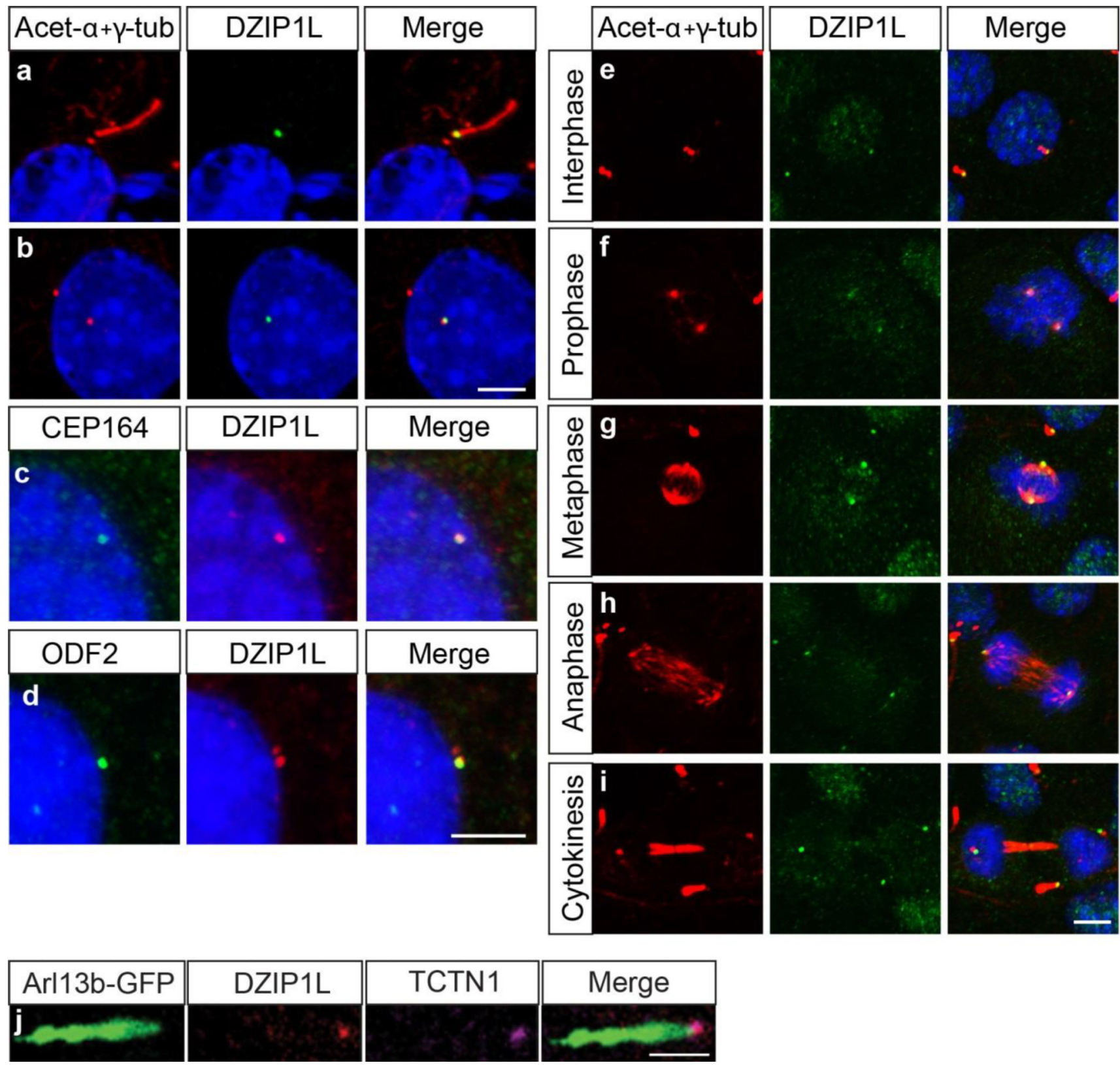

Figure 5.

Dzip11 is required for ciliary differentiation in the zebrafish kidney tubule. (a) Primary cilia (short arrows; myotome) and mono motile cilia (long arrows; pronephric duct) in a $24 \mathrm{hpf}$ wild-type embryo. (b) No obvious difference in primary (short arrows) and mono motile cilia (long arrows) differentiation in a dzip11 splice morpholino (e5i5) injected embryo at 24 hpf ( $\mathrm{n}=10)$. (c) MCC motile cilia bundles (arrows) in the pronephric duct of a wild-type embryo at $48 \mathrm{hpf}$ (arrows). (d) MCC motile cilia bundles (arrows) are reduced in a $48 \mathrm{hpf}$ maternal-zygotic dzip11 mutant zebrafish embryo $(n=8)$. (e) Quantification of MCC cilia bundles in wild-type and maternal-zygotic dzip11 mutant zebrafish embryos. 8 embryos from each group were analyzed. $* * \mathrm{p}=0.0017$ based on unpaired Student's $t$-test. Error bars show SEM. In all panels, cilia were stained with anti-acetylated tubulin antibody (magenta) and 
nuclei with DAPI (blue); in panels a,b,f-i cell membranes were stained with $\beta$-catenin antibodies (green). Scale bars in a,b,f-i $=10 \mu \mathrm{m} ; \mathrm{c}, \mathrm{d}=100 \mu \mathrm{m}$. 

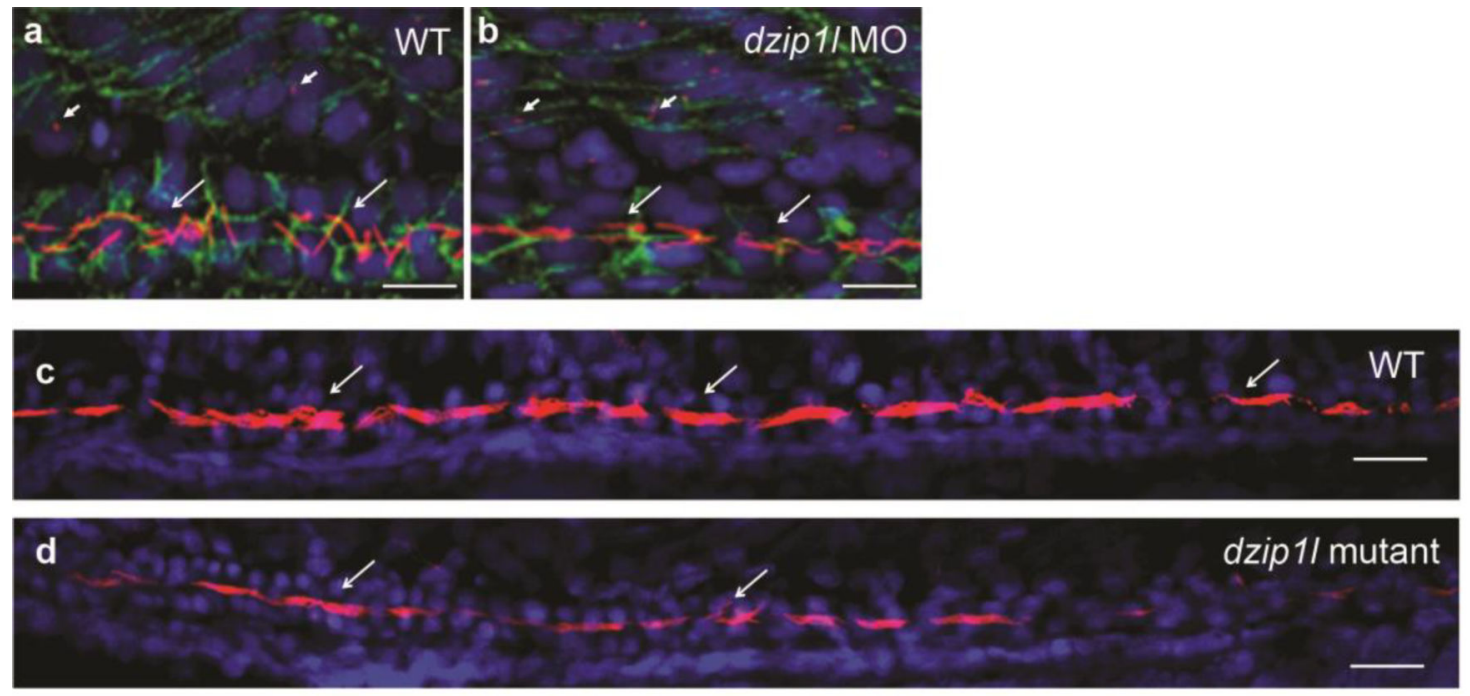

e

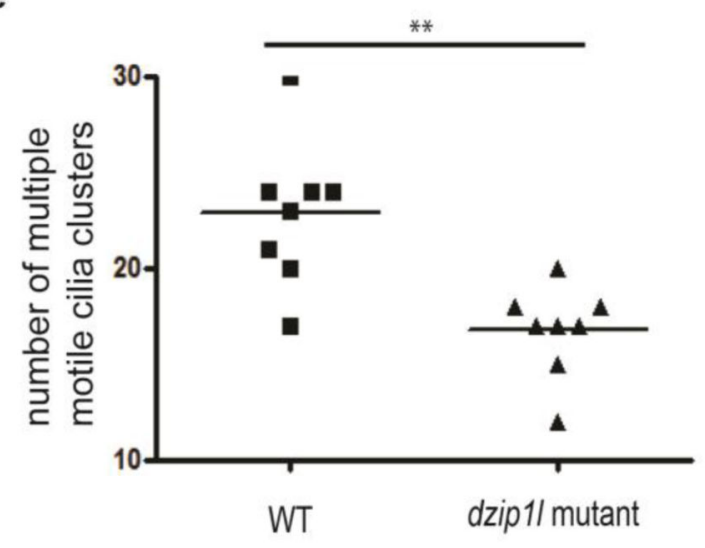

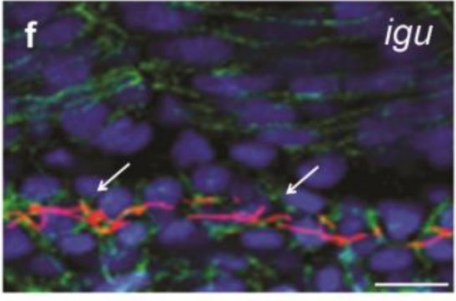

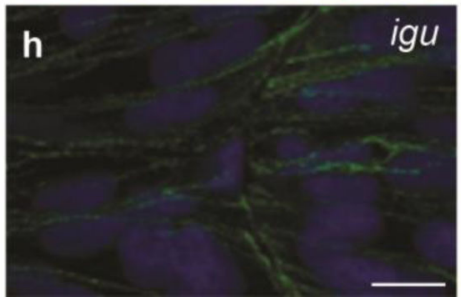

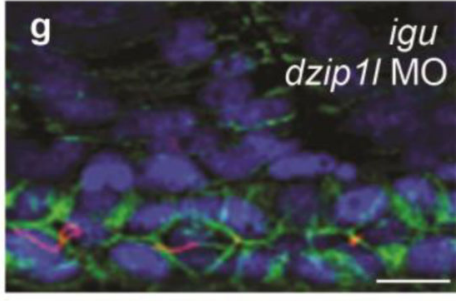

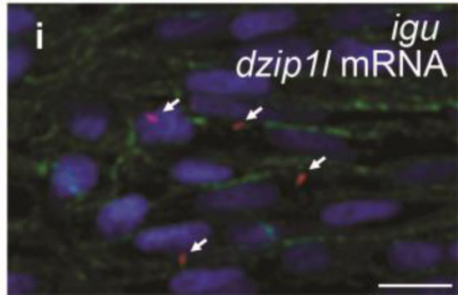

Figure 6.

DZIP1L associates with the ciliary transition zone protein SEPT2. (a) Schematic diagram of SEPT2, DZIP1L and a series of DZIP1L deletion mutants. (b) Interaction between SEPT2 (N-terminal FLAG) and DZIP1L and various DZIP1L deletion mutants (C-terminal HA) as determined by IP following transfection in HEK293T cells. (c,d) Endogenous interaction between DZIP1L and SEPT2 in RPE-1 cells. IP was performed with either the anti-DZIP1L antibody (Abnova; c) or the anti-SEPT2 antibody (d). (e) Expression of DZIP1L and SEPT2 in total cell lysate. (f) Co-localization of DZIP1L (cyan) and SEPT2 (magenta) in human dermal fibroblasts. ARL13B-GFP was used to mark the ciliary axoneme (green). (g,h) SEPT2 (green) localization to the transition zone was unaffected in DZIP1L mutant dermal fibroblasts from individual B155 (p.Gln155*). Ciliary axonemes and basal bodies were labelled with anti-acetylated tubulin and anti- $\gamma$-tubulin antibodies (magenta), respectively. Scale bars in $\mathrm{f}-\mathrm{h}=1 \mu \mathrm{m}$. All co-IP experiments were repeated at least three times. 


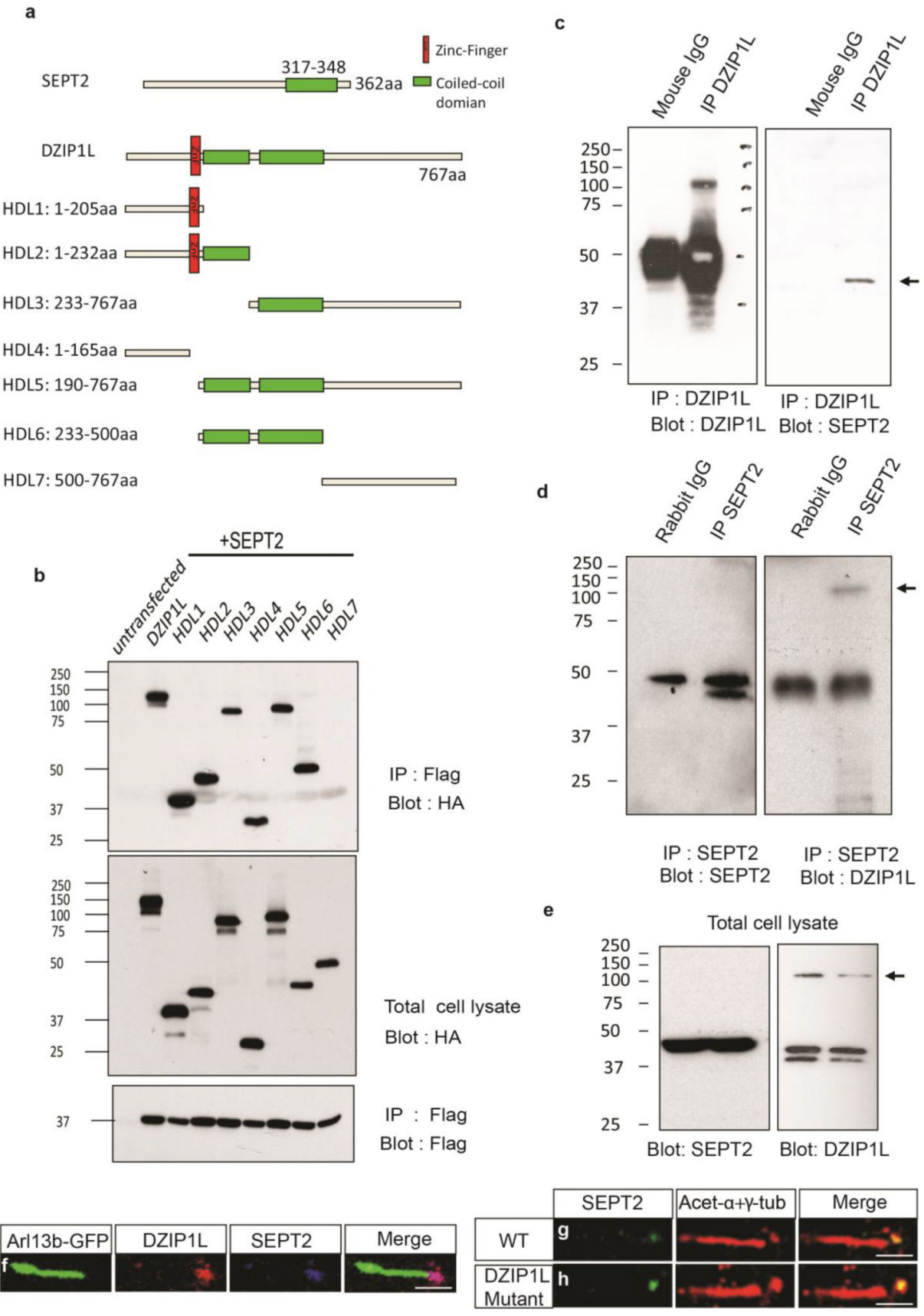

Figure 7.

Loss of DZIP1L affects the localization of PC1 and PC2 to the ciliary membrane. (a) In most wild-type ciliated MEFs PC1 (green; 7e12 mAb, Abcam) staining was distributed along the length of the axoneme as marked with ARL13B (magenta). (b) By contrast PC1 staining was more often concentrated at the proximal end of the axoneme in Dzip1/wpy/wpy MEFs (c) Quantification of the percentage of ciliated cells with PC1 along the axoneme $(n=6$, derived from $3 \mathrm{MEF}$ cell lines from independent embryos, each counted in two separate experiments; between 50-185 cells counted per replicate, total $737 \mathrm{Dzip} 1 \mathrm{I}^{+/+}$and 649 Dzip11 wpy/wpy cells counted). (d,e) Decreased PC1 (green) localization in the ciliary 
membrane in human dermal fibroblasts from affected individual B155 (p.Gln155*).

ARL13B (magenta) marks the ciliary axoneme. (g,h) Decreased PC2 localization (green) in the ciliary membrane in DZIPIL mutant human dermal fibroblasts. Acetylated-a-tubulin and $\gamma$-tubulin (magenta) mark the cilia. (f,i) Quantification of the percentage of ciliated cells with PC1 and PC2 along the axoneme, respectively, in control and DZIP1L mutant human dermal fibroblasts. For human cells, cilia were counted in three experiments, with cells from three independent coverslips counted for each experiment (approximately 100 ciliated cells counted on each coverslip). In all cases, quantification included cilia with or without detectable PC1 or PC2 staining. Statistical analyses based on unpaired Student's $t$-test. Error bars show SEM. For PC1 staining on MEFs, ****p<0.0001; PC1 and PC2 staining on human dermal fibroblasts, ${ }^{* * *} \mathrm{p}<0.001$. Scale bars in $\mathrm{a}, \mathrm{b}=2 \mu \mathrm{m} ; \mathrm{d}, \mathrm{e}, \mathrm{g}, \mathrm{h}=1 \mu \mathrm{m}$. 


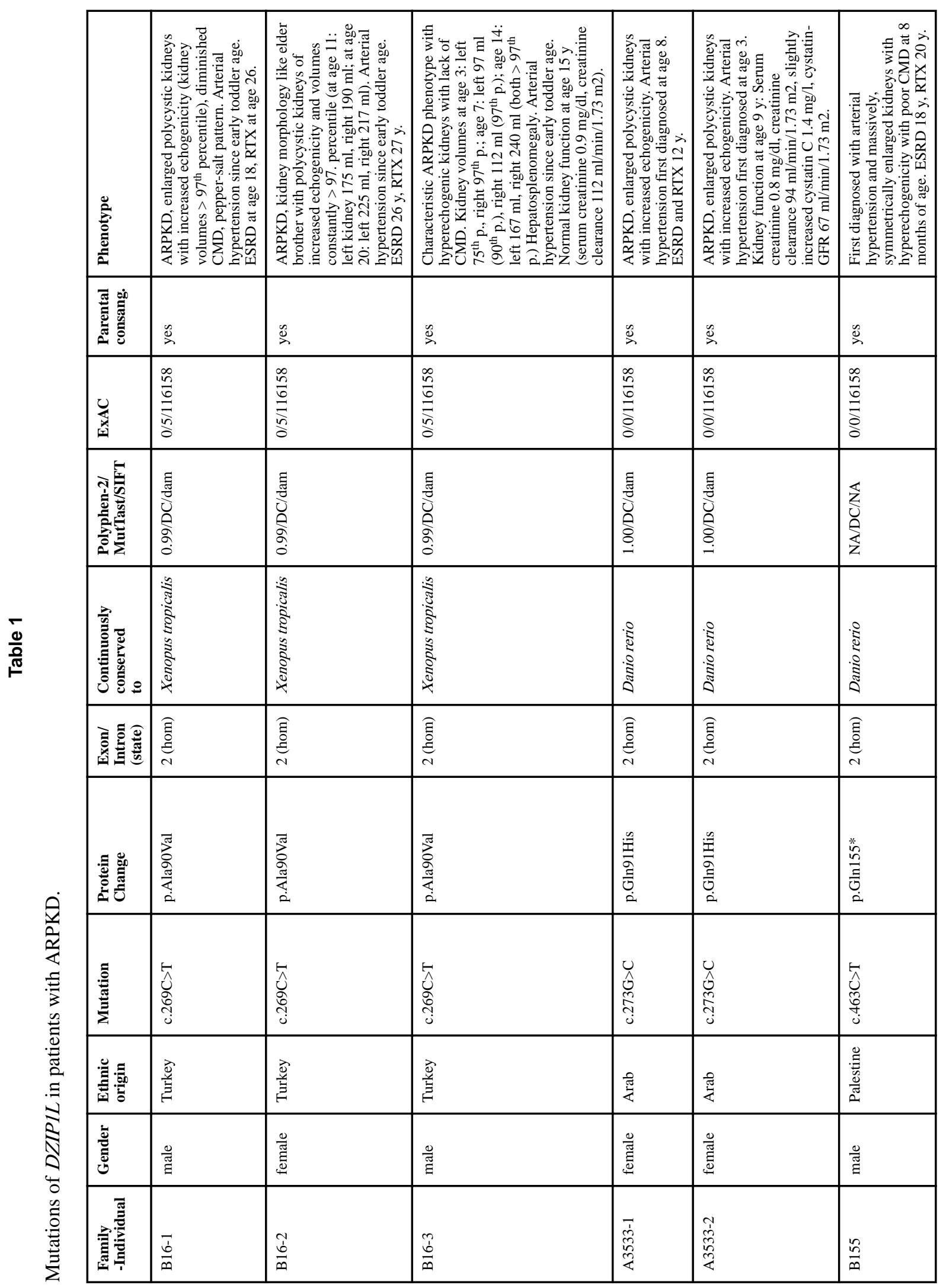

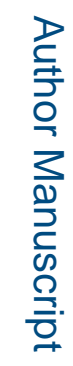

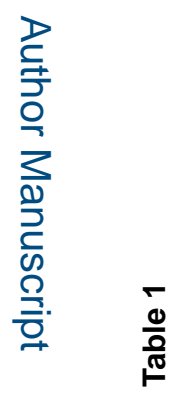

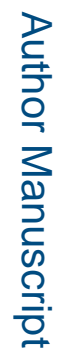

Nat Genet. Author manuscript; available in PMC 2018 January 01. 


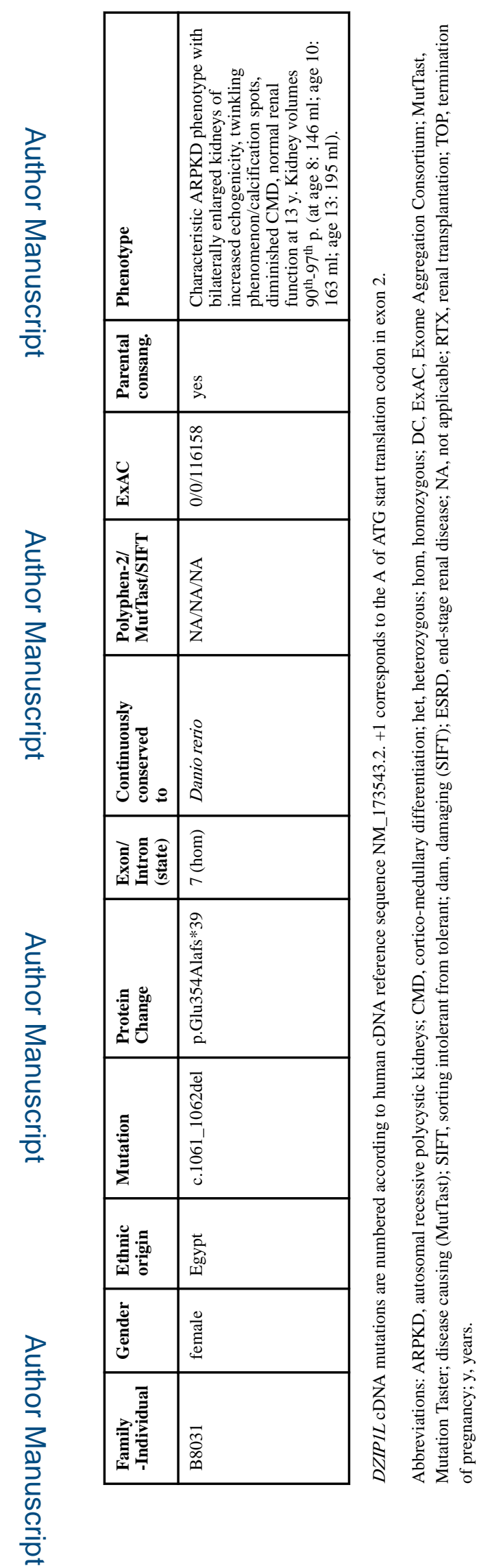

Nat Genet. Author manuscript; available in PMC 2018 January 01. 